\title{
Generalized Rings Around the McMullen Domain
}

\author{
Antonio Garijo*, HyeGyong Jang ${ }^{\dagger}$ \\ and \\ Sebastian M. Marotta
}

August 8, 2018

\begin{abstract}
We consider the family of rational maps given by $F_{\lambda}(z)=z^{n}+\lambda / z^{d}$ where $n, d \in \mathbb{N}$ with $1 / n+1 / d<1$, the variable $z \in \widehat{\mathbb{C}}$ and the parameter $\lambda \in \mathbb{C}$. It is known that when $n=d \geq 3$ there are infinitely many rings $\mathcal{S}^{k}$ with $k \in \mathbb{N}$, around the McMullen domain. The McMullen domain is a region centered at the origin in the parameter $\lambda$-plane where the Julia sets of $F_{\lambda}$ are Cantor sets of simple closed curves. The rings $\mathcal{S}^{k}$ converge to the boundary of the McMullen domain as $k \rightarrow \infty$ and contain parameter values that lie at the center of Sierpiński holes, i.e., open simply connected subsets of the parameter space for which the Julia sets of $F_{\lambda}$ are Sierpiński curves. The rings also contain the same number of superstable parameter values, i.e., parameter values for which one of the critical points is periodic and correspond to the centers of the main cardioids of copies of Mandelbrot sets. In this paper we generalize the existence of these rings to the case when $1 / n+1 / d<1$ where $n$ is not necessarily equal to $d$. The number of Sierpiński holes and superstable parameters on $\mathcal{S}^{1}$ is $\tau_{1}^{n, d}=n-1$, and on $\mathcal{S}^{k}$ for $k>1$ is given by $\tau_{k}^{n, d}=d n^{k-2}(n-1)-n^{k-1}+1$.
\end{abstract}

*Dep. d'Eng. Informàtica i Matemàtiques, Universitat Rovira i Virgili, Tarragona, Spain. Email: antonio.garijo@urv.cat

${ }^{\dagger}$ Faculty of Mathematics, University of Science Pyongyang D.P.R. of Korea.

$\ddagger$ Corresponding author: Sebastian M. Marotta, Department of Mathematics, Boston University, Boston, MA, USA. Email: smmarotta@gmail.com 


\section{Introduction}

In this paper we consider the family of complex rational maps ${ }^{1} F_{\lambda}: \widehat{\mathbb{C}} \rightarrow \widehat{\mathbb{C}}$ given by

$$
F_{\lambda}(z)=z^{n}+\frac{\lambda}{z^{d}}
$$

where $n, d \in \mathbb{N}$ with $1 / n+1 / d<1$ and the parameter $\lambda \in \mathbb{C}$. Let $m=n+d$ denote the degree of $F_{\lambda}$.

McMullen introduced $F_{\lambda}$ in [1] where he shows that when $\lambda \neq 0$ is sufficiently small, the Julia set of $F_{\lambda}$ is a Cantor set of simple closed curves, see also [2]. The condition $1 / n+1 / d<1$ is equivalent to $n d>m$ and this defines the set of values $n, d \geq 2$ with $m \geq 5$. The dynamics of some of these maps, the topological structures of their Julia sets and the structure of the parameter $\lambda$-planes have been widely studied by several authors, see for example $[2,3,4,5,6,7,8,9,10,11,12,13,14,15,16,17,18]$. A recent survey of results involving some of the maps in this family is given in [19]. The structure of the parameter planes of these maps is furthered studied in $[20,21,22]$.

In the dynamical plane the object of principal interest is the Julia set of $F_{\lambda}$, which we denote by $J\left(F_{\lambda}\right)$, and is the set of points at which the family of iterates $\left\{F_{\lambda}^{n}\right\}$ fails to be a normal family in the sense of Montel. It is known that $J\left(F_{\lambda}\right)$ is also the closure of the set of repelling periodic points for $F_{\lambda}$ as well as the boundary of the set of points whose orbits escape to $\infty$, or the set of points where the map presents chaotic behavior. For background results in complex dynamics see for example [23, 24, 25, 26, 27].

The function $F_{\lambda}$ has $2(n+d)-2$ critical points counted with multiplicity, namely, $n-1$ at $\infty, d-1$ at 0 and $n+d$ additional critical points whose orbits depend on the value of the parameter $\lambda$. One of the reasons this family of maps has gained so much attention is the fact that these "free" critical points all behave symmetrically. This implies that there is essentially one critical orbit and then the $\lambda$-plane is a natural parameter plane for each of these families.

Since $n \geq 2$, the point at $\infty$ is a superattracting fixed point for any value of $\lambda$. Let $B_{\lambda}$ denote the immediate basin of attraction of $\infty$, and notice that $B_{\lambda}$ is mapped to itself at least in an $n$-to- 1 fashion. When all the critical points are in $B_{\lambda}$ the Julia set of the map is a Cantor set of points

\footnotetext{
${ }^{1}$ We use $\mathbb{C}$ for the complex plane and $\widehat{\mathbb{C}}=\mathbb{C} \cup\{\infty\}$ for the Riemann sphere.
} 
and $B_{\lambda}$ is mapped to itself in an $m$-to- 1 fashion. Let $T_{\lambda}$ be the preimage of $B_{\lambda}$ that contains the origin. When $T_{\lambda}$ is disjoint from $B_{\lambda}$ then $T_{\lambda}$ is a simply connected set that is mapped $d-$ to- 1 onto $B_{\lambda}$. We call $T_{\lambda}$ the trap door, since every point that escapes to infinity and it is not in $B_{\lambda}$ must fall throuth $T_{\lambda}$ along its orbit.

When the orbits of all the critical points of $F_{\lambda}$ are attracted to $\infty$, the Julia set of $F_{\lambda}$ can have exactly three different topological structures. The following result is included in [2], where a more general result is proved.

Theorem 1.1 (The Escape Trichotomy). Fix $n, d \in \mathbb{N}$ with $1 / n+1 / d<1$ then,

(a) If the critical values of $F_{\lambda}$ lie in $B_{\lambda}$, then the Julia set is a Cantor set of points.

(b) If the critical values of $F_{\lambda}$ lie in $T_{\lambda} \neq B_{\lambda}$, then the Julia set is a Cantor set of simple closed curves.

(c) If the critical values of $F_{\lambda}$ lie in any other preimage of $T_{\lambda}$, then the Julia set is a Sierpiński curve.

Case 1 corresponds to $\lambda$ in the Cantor set locus, that is, an open connected set that surrounds infinity in the $\lambda$-plane where the Julia set of $F_{\lambda}$ is a Cantor set of points. In this case the Fatou set consists of one infinitely connected region, i.e., $B_{\lambda}=T_{\lambda}$. Case 2 corresponds to the McMullen domain, that is, a punctured (at the origin) open disk that is bounded by a simple closed curve in the $\lambda$-plane where all the maps $F_{\lambda}$ have Julia sets that are Cantor sets of simple closed curves. Then the Fatou set consists of 2 simply connected domains $\left(B_{\lambda}\right.$ and $\left.T_{\lambda}\right)$ and infinitely many concentric annuli that are preimages of $T_{\lambda}$. Case 3 is very different; the parameter plane of the family $F_{\lambda}$ shows infinitely many Sierpinski holes, that is, disjoint simply connected domains with parameters for which the Julia set of $F_{\lambda}$ is a Sierpiński curve. A Sierpiński curve is a set homeomorphic to the well-known Sierpiński carpet fractal, see [28]. For convenience, when comparing different maps from the family $F_{\lambda}$, sometimes we use $\langle n, d\rangle$ to denote $z^{n}+\lambda / z^{d}$. Figure 1 illustrates the Escape Trichotomy Theorem 1.1. Unlike cases 1 and 2, Sierpiński curve Julia sets happen also when the free critical points do not escape to infinity, see for example [29, 30, 31].

In this paper we discuss some of the properties of the parameter plane for these maps. The following result can be found in [6]. 


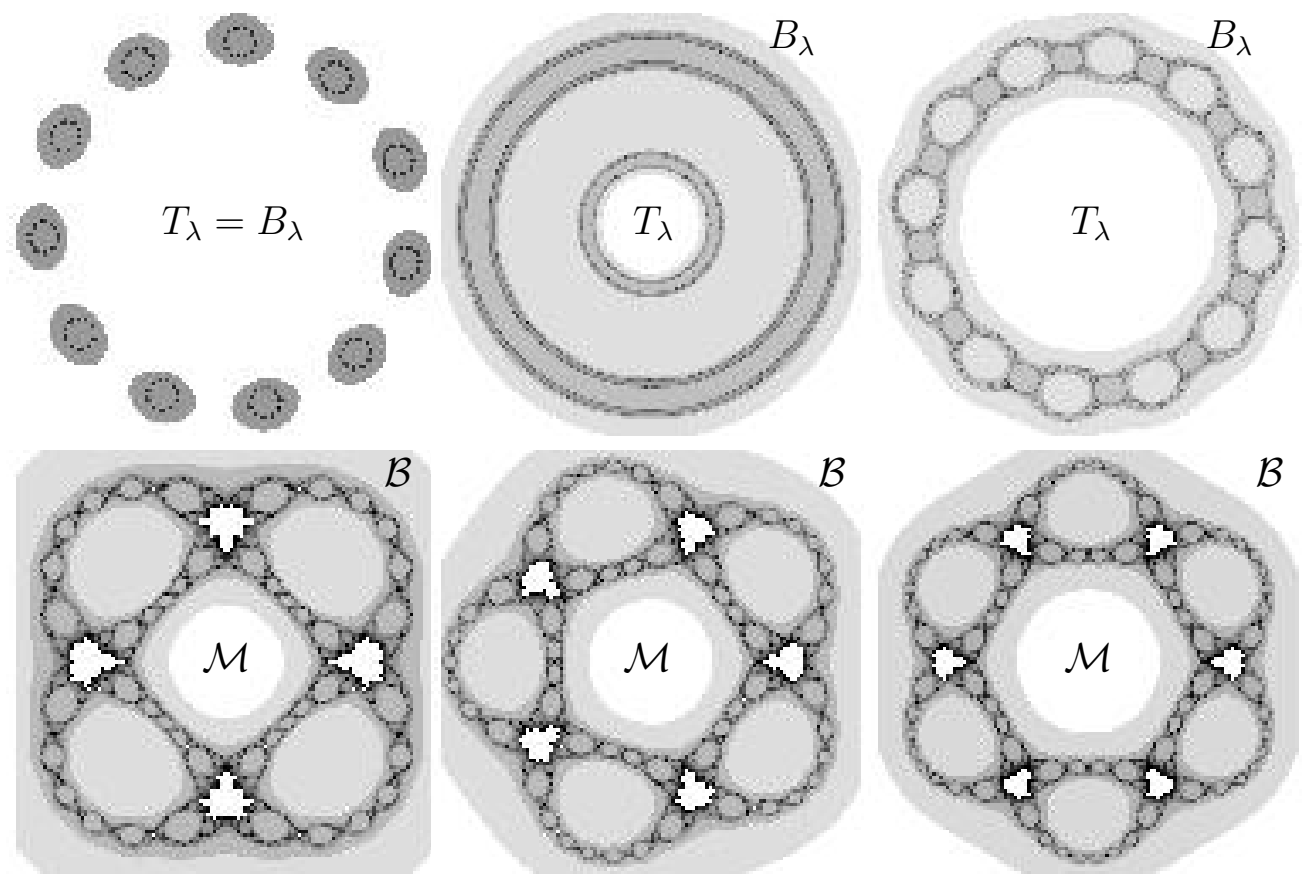

Figure 1: The top line of pictures shows Julia sets for the family $F_{\lambda}(z)$ that illustrate the three topological structures presented in the Escape Trichotomy Theorem 1.1. From left to right: a Cantor set of points for the case < $n, d>=<5,6>$ with $\lambda=1+i$, a Cantor set of simple closed curves for the case $\langle 6,5\rangle$ with $\lambda=0.001(1+2 i)$ and a Sierpiński curve for the case $\left\langle 7,5>\right.$ with $\lambda=13 i / 50$. The basin of attraction of $\infty$ is $B_{\lambda}$ and its preimage containing the origin is the trap door $T_{\lambda}$. The maps $<5,6>$ and $<6,5>$ have degree $m=11$ and therefore there are 11 sectors of the sphere that are equal to each other under rotation by $2 \pi / 11$. The map $<7,5>$ has degree $m=12$ and therefore there are 12 sectors of the sphere that are equal to each other under rotation by $\pi / 6$. The bottom line of pictures shows (from left to right) the parameter $\lambda$-planes for $\langle 5,6\rangle,\langle 6,5\rangle$ and $<7,5>$. Notice the $(n-1)$-fold symmetry of these planes illustrated by the white baby Mandelbrot sets symmetrically distributed around the origin $\lambda=0$. The unbounded region is the Cantor set locus $\mathcal{B}$, the disk centered at the origin is the McMullen domain $\mathcal{M}$ and the other shaded disks in the connectedness locus correspond to Sierpiński holes. 
Theorem 1.2 (Principal baby Mandelbrot sets with halos) Fix $n, d \in \mathbb{N}$ with $1 / n+1 / d<1$, then there exists a small copy of the Mandelbrot set in parameter $\lambda$-plane for $F_{\lambda}$ in each of the $n-1$ sectors of the form

$$
\frac{(2 j-1) \pi}{n-1}<\operatorname{Arg} \lambda<\frac{(2 j+1) \pi}{n-1}, \quad j=0,1,2, \ldots, n-2 .
$$

Each of these baby Mandelbrot sets have infinitely many "halos" attached, i.e., infinitely many points on the boundary of Sierpinki holes.

Notice that the cases with $n=2$ and $d>2$ show only one principal baby Mandelbrot set straddling the real axis. These babies are slightly different from the ones in the rest of the family in the sense that the "tail" (the portion of the set to the left of the period-2 bulb) of these Mandelbrot sets and the period-2 bulbs are smaller. When $n=d=2$, a map that is not in our family, the tail seems to completely disappear. See Figure 2.

It is known that there are infinitely many disjoint Sierpiński holes for each of these families (see, [10] and [32]) and that there is a unique parameter in each Sierpiński hole for which the orbit of the critical point lands on 0 at some iteration and therefore on $\infty$ at the next iteration, say at iteration $k>2$. We then call this $\lambda$-value the center of the Sierpiński hole and $k$ the escape time of the hole. All other parameters in a given Sierpiński hole have the property that the orbit of the critical value lands in $B_{\lambda}$ at the escape time iterate. By Whyburn's result, the Julia sets corresponding to any two parameters drawn from a Sierpiński hole are homeomorphic. However, as shown in [2], there exist Sierpiński holes corresponding to each escape time $k \geq 3$, and these have the property that if $\lambda_{1}$ and $\lambda_{2}$ lie in Sierpiński holes with different escape times, then $F_{\lambda_{1}}$ and $F_{\lambda_{2}}$ are not topologically conjugate on their Julia sets. Much more information about the $\lambda$-planes for the case $n=d \geq 3$ is given in $[20,21,22]$.

Our goal in this paper is to investigate further properties of the parameter plane for these maps and, in particular, to extend a result from [3] that explains the structure of the parameter plane in a neighborhood of the McMullen domain.

Figure 3 displays magnifications of the region around the McMullen domain in the case $n=5$ and $d=6$. In the first image, note that there are four large Sierpiński holes symmetrically placed around the McMullen domain. These Sierpiński holes all have escape time 3. Between the Sierpiński holes 

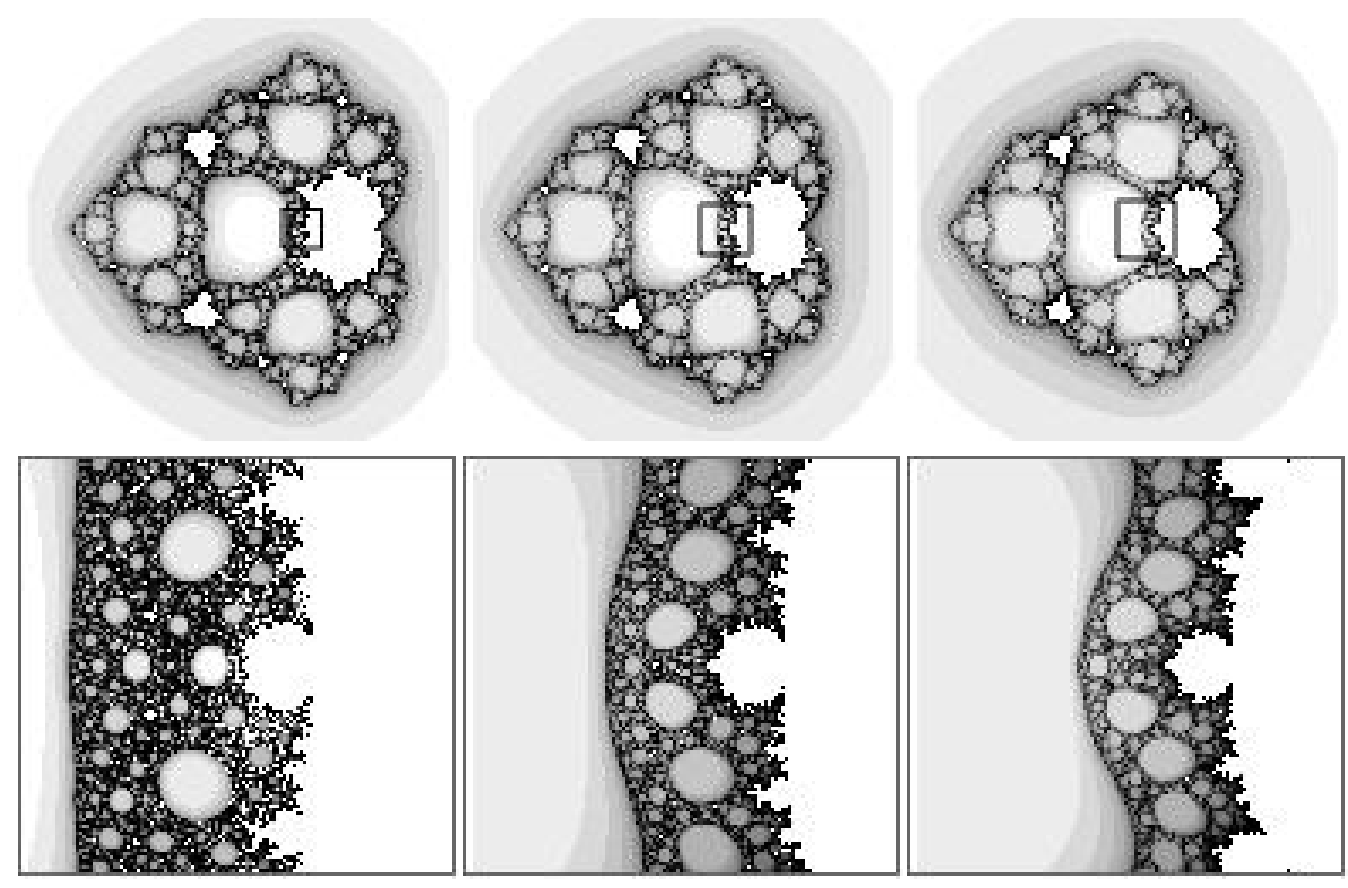

Figure 2: The top line of pictures shows the parameter planes for the family $z^{n}+\lambda / z^{d}$ from left to right we have $<n, d>=<2,2>,<2,3>$ and $<2,4>$. The bottom line of pictures shows magnifications in the region $-0.03<\Re e \lambda<0.03$ and $-0.03<\Im m \lambda<0.03$.

there are 4 small copies of the Mandelbrot set. Indeed, one may draw a simple closed curve, $\mathcal{S}^{1}$, that encircles the McMullen domain and passes through the centers of each of these Sierpiński holes and the centers of the main cardioids of the Mandelbrot sets. That is, on this simple closed curve, we find four parameter values for which $F_{\lambda}$ has a superstable fixed point and four other values for which $F_{\lambda}$ maps the critical points to $\infty$, and these parameter values alternate between the superstable and the centers of Sierpiński holes as the parameter winds around the closed curve.

Inside these 4 Sierpiński holes there is another simple closed curve, $\mathcal{S}^{2}$, passing through the centers of 20 Sierpiński holes. Each of these holes has escape time 4. Also, each pair of these holes apparently has either a small copy of a Mandelbrot set or a portion of a principal Mandelbrot set between them. Examining the further magnification in Figure 3, we see a smaller 

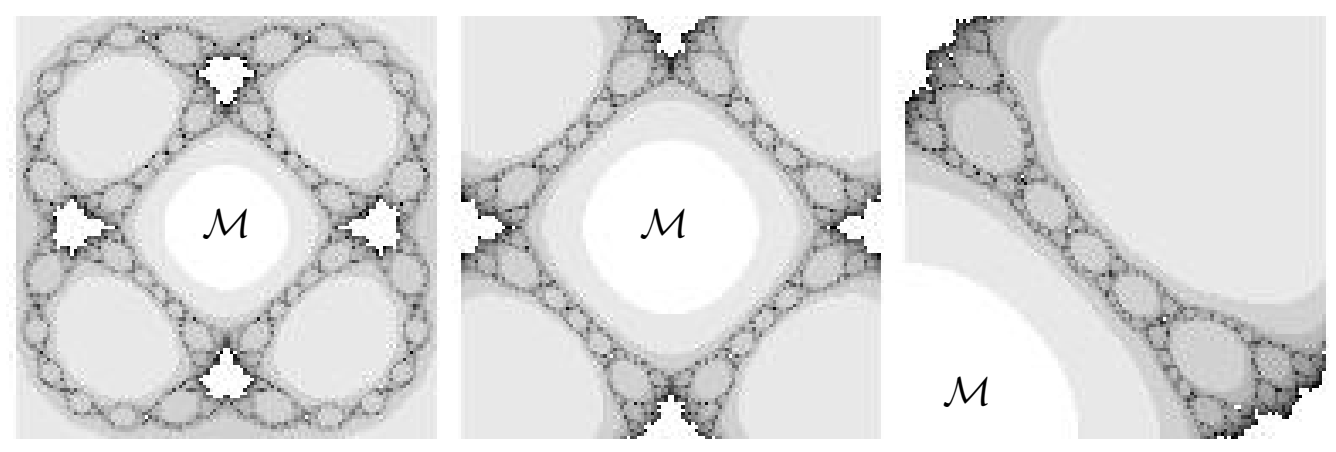

Figure 3: The parameter plane for the family $z^{5}+\lambda / z^{6}$ and magnifications that show the structure around the McMullen domain.

closed curve $\left(\mathcal{S}^{3}\right)$ passing through 96 Sierpiński holes with escape time 5 and, inside that curve, an even smaller curve $\left(\mathcal{S}^{4}\right)$ passing through 476 Sierpiński holes with escape time 6 . We call these curves rings around the McMullen domain.

The general formula for the case $n=d \geq 3$ gives $\tau_{k}^{n}=(n-2) n^{k-1}+1$ Sierpinski holes for $k \in \mathbb{N}$, see [3]. The proof in [3] works for the cases where $n=d \geq 5$ and special corrections need to be made for $n=d=3$ and 4 , see [3]. In a subsequent paper [13] it was shown that these superstable parameter values each lie at the center of a small copy of a Mandelbrot set.

Our goal is to extend these results by proving the existence of the rings $\mathcal{S}^{k}$ and presenting the general formula when $1 / n+1 / d<1$ and $n$ is not necessarily equal to $d$. Our proof works for $n, d \geq 5$ and special corrections need to be made for the other cases. In this paper we prove the following theorem.

Theorem A. (Generalized Rings Around the McMullen Domain) Let $n, d \geq 5$ then the McMullen domain for the family $F_{\lambda}(z)=z^{n}+\lambda / z^{d}$ is surrounded by infinitely many simple closed curves $\mathcal{S}^{k}$ for $k=1,2, \ldots$ having the property that:

(a) Each curve $\mathcal{S}^{k}$ surrounds the McMullen domain as well as $\mathcal{S}^{k+1}$, and the $\mathcal{S}^{k}$ accumulate on the boundary of the McMullen domain as $k \rightarrow \infty$;

(b) The curve $\mathcal{S}^{k}$ meets the centers of $\tau_{k}^{n, d}$ Sierpinski holes, each with escape time $k+2$ where, for $k=1$ we have $\tau_{k}^{n, d}=n-1$, and for $k>1$,

$$
\tau_{k}^{n, d}=d n^{k-2}(n-1)-n^{k-1}+1 .
$$


(c) The curve $\mathcal{S}^{k}$ also passes through $\tau_{k}^{n, d}$ superstable parameter values where a critical point of $F_{\lambda}$ is periodic of period $q k$ for some $q \in \mathbb{N}$.

The ideas behind the proof are similar to the ones used in [3]. However, some important differences are the fact that in the general case there is no involution of the form $z \mapsto \lambda^{1 / n} / z$ that conjugates $F_{\lambda}$ to itself as in the case $n=d$. Also, the proof in the more general case requires the use of generalized symmetries and ideas that where developed in $[6,33]$.

The paper is organized as follows. In $\S 2$ we explain the main dynamical properties of the family $F_{\lambda}$ and describe part of the general structure of the parameter $\lambda$-plane. In $\S 3$ we present the proof of Theorem A. In $\S 3.1$ we prove the base case, that is, the existence of the curve $\mathcal{S}^{1}$. In $\S 3.2$ and $\S 3.3$ we present the mapping properties of $F_{\lambda}$. The existence of the rest of the rings $\mathcal{S}^{k}$ for $k>1$ is proven in $\S 3.4$ and $\S 3.5$. The rings are first constructed in the dynamical plane $(\S 3.4)$ and then translated to the parameter plane $(\S 3.5)$.

\section{Preliminaries and previous results}

In this section we explain the main properties that arise in the dynamical and the parameter planes of the family $F_{\lambda}$, given by

$$
F_{\lambda}(z)=z^{n}+\frac{\lambda}{z^{d}}
$$

where $n, d \in \mathbb{N}$ with $n, d \geq 2$ and the parameter $\lambda \in \mathbb{C}$.

\subsection{Dynamical plane}

Note that when $\lambda \neq 0, F_{\lambda}$ has degree $m=n+d$ and so it has $2 m-2$ critical points counting multiplicities. Infinity and the origin are critical points with multiplicities $n-1$ and $d-1$, respectively. As we mentioned before, $\infty$ is a superattracting fixed point of $F_{\lambda}$ and 0 is the only finite preimage of it. Thus, the remaining $m$ "free" critical points, are given by

$$
c_{\lambda}=\left(\frac{\lambda d}{n}\right)^{1 / m}
$$

We see that the critical points all lie on a circle, the critical circle, and are symmetrically distributed around the origin. The associated critical values 
$v_{\lambda}=F_{\lambda}\left(c_{\lambda}\right)$ are also distributed in a circle, the critical value circle, and simple computations show that

$$
v_{\lambda}=F_{\lambda}\left(c_{\lambda}\right)=\frac{m}{d}\left(\frac{\lambda d}{n}\right)^{n / m} .
$$

For later reference, we also point out that there are $m$ prepoles $p_{\lambda}$, verifying $F_{\lambda}\left(p_{\lambda}\right)=0$, so

$$
p_{\lambda}=(-\lambda)^{1 / m}
$$

that lie on a circle, the prepole circle, and are also symmetrically located around the origin.

We use $C_{\lambda}, V_{\lambda}$ and $P_{\lambda}$ to denote the critical circle, the critical value circle and the prepole circle, respectively. The three circles are centered at the origin with radius $\left|c_{\lambda}\right|,\left|v_{\lambda}\right|$ and $\left|p_{\lambda}\right|$, respectively. We can compare any two of these three circles in the natural way by comparing their corresponding radii. We introduce the following notation,

- $C_{\lambda} \prec V_{\lambda}$ if and only if $\left|c_{\lambda}\right|<\left|v_{\lambda}\right|$,

- $C_{\lambda}=V_{\lambda}$ if and only if $\left|c_{\lambda}\right|=\left|v_{\lambda}\right|$,

- $C_{\lambda} \succ V_{\lambda}$ if and only if $\left|c_{\lambda}\right|>\left|v_{\lambda}\right|$.

When the context is clear we may drop the subindex $\lambda$ of the critical points, critical values and prepoles. Let $\nu=e^{i \frac{2 \pi}{m}}$ be a primitive $m$-th root of unity, we write $c_{j}=\nu^{j} c_{\lambda}$ for $j=0,1, \cdots, m-1$, where $c_{\lambda}$ is a concrete critical point. The same applies to the critical values and the prepoles. We restrict the use of the letters $c, v$ and $p$ for critical points, critical values and prepoles, respectively.

We turn our attention to the symmetries that arise in the dynamical plane of the family $F_{\lambda}$. It is easy to check that $F_{\lambda}(\nu z)=\nu^{n} F_{\lambda}(z)$ and then for all $k \in \mathbb{N}$,

$$
F_{\lambda}^{k}(\nu z)=\nu^{n^{k}} F_{\lambda}^{k}(z)
$$

Hence the orbits of points of the form $\nu^{j} z$ all behave "symmetrically" under iteration of $F_{\lambda}$. For example, if $F_{\lambda}^{k}(z) \rightarrow \infty$, then $F_{\lambda}^{k}\left(\nu^{j} z\right)$ also tends to $\infty$ for each $j$. If $F_{\lambda}^{k}(z)$ tends to an attracting cycle, then so does $F_{\lambda}^{k}\left(\nu^{j} z\right)$. Note, however, that the cycles involved may be different depending on $j$ and, indeed, they may even have different periods. Nonetheless, all points lying on these attracting cycles are of the form $\nu^{j} z_{0}$ for some $z_{0} \in \mathbb{C}$. 
Theorem 2.1 (Symmetries in dynamical plane, see [6]) Let $n, d \in \mathbb{N}$ with $1 / n+1 / d<1$ and let $\nu=e^{i \frac{2 \pi}{m}}$, then there exist $r, q \in \mathbb{N}$ such that for all $k \geq r$

$$
F_{\lambda}^{q+k}(\nu z)=\nu^{n^{k}} F_{\lambda}^{q+k}(z) .
$$

Moreover,

(a) If every prime factor of $m$ is a prime factor of $n$, then there exists $r \in \mathbb{N}$ such that $\forall k \geq r, \nu^{n^{k}}=1$ and the orbits of $\nu z$ and $z$ coincide, that is, $F_{\lambda}^{k}(\nu z)=F_{\lambda}^{k}(z)$.

(b) If $m$ and $n$ are relatively prime, then there exists $q \in \mathbb{N}$ such that $\nu^{n^{q}}=$ $\nu$ and $F_{\lambda}^{q}$ is conjugate to itself under $z \mapsto \nu z$, that is, $F_{\lambda}^{q}(\nu z)=\nu F_{\lambda}^{q}(z)$.

This implies that the dynamics and the Julia sets of $F_{\lambda}$ are symmetric under rotation by $2 \pi / m$, see Figure 1 .

We call the straight line connecting the origin to $\infty$ and through one of the critical points (resp., prepoles) a critical point ray (resp., prepole ray). The critical point rays $C_{j}$ are defined by $t c_{j}$ with $0<t<\infty$ and $j=$ $0,1, \ldots, m-1$. It follows from Equation (2.4) that for all $j \in \mathbb{N}$ we have $F_{\lambda}\left(\nu^{j} z\right)=\nu^{j n} F_{\lambda}(z)$ and then the image of the ray $C_{j}$ is given by $F_{\lambda}\left(t c_{j}\right)=$ $t^{\prime} v_{j^{\prime}}$, where $v_{j^{\prime}}=F_{\lambda}\left(c_{j}\right)$ and $j^{\prime}=j n(\bmod m)$ and $t^{\prime}>0$.

Similarly, let the prepole ray $P_{j}$ be given by $t p_{j}$ with $0<t<\infty$ and $j=0,1, \ldots, m-1$. Then the image of the ray $P_{j}$ is given by $F_{\lambda}\left(t p_{j}\right)=t^{\prime} p_{j}^{n}$, where $t^{\prime}=t^{-d}\left(t^{m}-1\right)$ is a real number. Notice that if $t>1$, then $t^{\prime}>0$, and if $t<1$, then $t^{\prime}<0$. It follows that the straight ray connecting prepole $p_{j}$ to $\infty$ is mapped onto a straight ray connecting the origin to $\infty$, and the segment connecting the origin and the prepole $p_{j}$ is mapped onto the opposite straight ray to that of $t>1$.

Hence each of the critical point rays is mapped in a two-to-one fashion onto a ray connecting $v_{j^{\prime}}$ to $\infty$. Similarly, each of the $m$ prepole rays is mapped in a one-to-one fashion onto the straight line defined by $t^{\prime} p_{j}^{n}$, where $t^{\prime}$ is now any real number.

\subsection{Parameter plane}

Let $\mathcal{C}$ denote the connectedness locus, that is, the set of $\lambda$-values for which the Julia set of $F_{\lambda}$ is a connected set. The complement of this set in $\mathbb{C}^{*}=\mathbb{C}-\{0\}$ has two components each homeomorphic to a disk with a puncture, namely, 
the Cantor set locus, that is the set of $\lambda$-values for which the Julia set is a Cantor set of points, that we denote by $\mathcal{B}$; and the McMullen domain, that is, the set of $\lambda$-values for which the Julia set is a Cantor set of simple closed curves, that we denote by $\mathcal{M}$. See Figures 1 and 3 . in $[6]$,

The following bounds of the regions $\mathcal{C}, \mathcal{B}$ and $\mathcal{M}$ in the $\lambda$-plane are found

$$
\begin{array}{ll}
\text { If } \lambda \leq \lambda_{\min }=\frac{n}{d}\left(\frac{d}{2 m}\right)^{\frac{m d}{n d-m}} & \text { then } \lambda \in \mathcal{M} . \\
\text { If } \lambda \geq \lambda_{\max }=\frac{n}{d}\left(\frac{2 d}{m}\right)^{\frac{m}{n}} & \text { then } \lambda \in \mathcal{B} .
\end{array}
$$

We turn now our attention to the relative position of the three concentric circles defined in the dynamical plane: the critical circle $C_{\lambda}$, the critical value circle $V_{\lambda}$ and the prepole circle $P_{\lambda}$. The relative position between these circles depends on the values of $n, d$ and $\lambda$. We first analyze the relative position between $C_{\lambda}$ and $P_{\lambda}$, since it does not depend on $\lambda$. Thus, for example when $n=d$ then $P_{\lambda}=C_{\lambda}$, when $n>d$ then $P_{\lambda} \succ C_{\lambda}$ and, finally when $n<d$ then $P_{\lambda} \prec C_{\lambda}$.

Finally, we also define two auxiliary real values $\lambda_{c}$ and $\lambda_{p}$. The first one, $\lambda_{c}$, is the real value of $\lambda$ such that $V_{\lambda}=C_{\lambda}$. The second one, $\lambda_{p}$, is such that $V_{\lambda}=P_{\lambda}$. Simple computations show that

$$
\lambda_{c}=\frac{n}{d}\left(\frac{d}{m}\right)^{\frac{m}{n-1}} \text { and } \lambda_{p}=\left(\frac{d}{m}\right)^{\frac{m}{n-1}}\left(\frac{n}{d}\right)^{\frac{n}{n-1}} .
$$

The circle of radius $\lambda_{c}$ plays an important role in the parameter plane, for if $\lambda$ lies inside the circle of radius $\lambda_{c}$, that is, $|\lambda|<\lambda_{c}$, then $V_{\lambda} \prec C_{\lambda}$. Instead, if $\lambda$ lies in the circle of radius $\lambda_{c}$, that is, $|\lambda|=\lambda_{c}$, then $V_{\lambda}=C_{\lambda}$. Finally, if $|\lambda|>\lambda_{c}$ then $V_{\lambda} \succ C_{\lambda}$. We call the circle of radius $\lambda_{c}$ in parameter plane the dividing critical circle.

In a similar fashion, if $\lambda$ lies inside the circle of radius $\lambda_{p}$ then $V_{\lambda} \prec P_{\lambda}$. We call the circle of radius $\lambda_{p}$ in parameter plane the dividing prepole circle.

It is easy to see that if $n>d$ then $\lambda_{p}>\lambda_{c}$ and this implies that $P_{\lambda} \succ C_{\lambda}$, that is, the prepole circle lies outside the critical point circle. If $n=d$ then $\lambda_{p}=\lambda_{c}$ and $P_{\lambda}=C_{\lambda}$. Finally, if $d>n$ then $\lambda_{c}>\lambda_{p}$ and $C_{\lambda} \succ P_{\lambda}$.

The parameter plane also possesses several symmetries. First of all, we have $\overline{F_{\lambda}(z)}=F_{\bar{\lambda}}(\bar{z})$ so that $F_{\lambda}$ and $F_{\bar{\lambda}}$ are conjugate via the map $z \mapsto \bar{z}$. Therefore the parameter plane is symmetric under the map $\lambda \mapsto \bar{\lambda}$. We also have $(n-1)$-fold symmetry in the parameter plane for $F_{\lambda}$. See Figure 1. 
Theorem 2.2 (Symmetries in parameter $\lambda$-plane, see [6]) Let $n, d \in \mathbb{N}$ with $1 / n+1 / d<1$. Let $\omega=e^{i \frac{2 \pi}{n-1}}$, then there are $k^{\prime}, q \in \mathbb{N}$ such that $F_{\lambda}^{q}$ is conjugate to $F_{\omega \lambda}^{q}$ under $z \mapsto \omega^{\frac{k^{\prime}}{q}} z$, that is,

$$
F_{\omega \lambda}^{q}\left(\omega^{\frac{k^{\prime}}{q}} z\right)=\omega^{\frac{k^{\prime}}{q}} F_{\lambda}^{q}(z)
$$

It follows that the parameter $\lambda$-plane is symmetric under the map $\lambda \mapsto \omega \lambda$.

\section{Proof of Theorem A}

In this section we prove the main result of this paper. The proof of the existence of the rings $\mathcal{S}^{k}$ for $k \in \mathbb{N}$ when $n, d \in \mathbb{N}$, and verifying $n, d \geq 5$, is done by induction.

\subsection{The curve $\mathcal{S}^{1}$}

In the case that $n=d$ the first ring $\mathcal{S}^{1}$, is a round circle in the parameter plane such that for all $\lambda$ values in this circle, the critical points, the critical values and the prepoles belong to the same circle. Since, when changing the argument of $\lambda$, the critical points and prepoles rotate at a rate $n$-times slower than the critical value, we get the results of [3].

In the case that $n \neq d$ the critical circle $C_{\lambda}$ and the prepole cicle $P_{\lambda}$ have different radii, and this fact makes the proof of $\mathcal{S}^{1}$ necessary. However, in this section we show that there is a natural curve, denoted by $\Gamma_{\lambda}$, in the dynamical plane that goes through each critical point and prepole of $F_{\lambda}$. See Figure 5. We translate this curve $\Gamma_{\lambda}$ to the parameter plane to find the curve $\mathcal{S}^{1}$ that goes through the centers of Sierpinski holes and the centers of the main cardioids of the principal baby Mandelbrot sets.

We split the proof of the existence of the curve $\mathcal{S}^{1}$ in the parameter plane into two parts. In the first part, Propositions 3.1, 3.2 and 3.3, we prove the existence of a Jordan curve $\Gamma_{\lambda}$ that surrounds the origin in dynamical plane such that it contains every critical point and prepole and is symmetric under rotation by $2 \pi / m$. We also show that the curve $\Gamma_{\lambda}$ is contained in between the critical circle $C_{\lambda}$ and the prepole circle $P_{\lambda}$, except at the intersection points where they agree. In the second part, Proposition 3.4, we transfer this curve to the parameter $\lambda$-plane, using the Schwarz Lemma, to show the existence of $\mathcal{S}^{1}$. This new curve is also a Jordan curve that surrounds the 
origin and contains the centers of the main cardioids of $(n-1)$ principal baby Mandelbrot sets and the same number of centers of Sierpinski holes in the case $n=d$. Indeed, the existence of these $(n-1)$ principal baby Mandelbrot set was proved in [6].

We recall that $V_{\lambda}$ denotes the critical value circle, that is the circle of radius $\left|v_{\lambda}\right|$ centered at the origin.

Proposition 3.1 The preimage of the critical value circle $V_{\lambda}$ consists of two simple closed curves $\Gamma_{I}$ and $\Gamma_{E}$ that surround the origin and intersect exactly at the $m$ critical points. There is a third simple closed curve $\Gamma_{\lambda}$ that goes through the critical points and prepoles and is contained in between $\Gamma_{I}$ and $\Gamma_{E}$. Moreover, $F_{\lambda}$ maps the curve $\Gamma_{\lambda}$ inside the closed disk bounded by $V_{\lambda}$ and for all $z \in \Gamma_{\lambda}$ we have that

$$
\min \left\{\left|c_{\lambda}\right|,\left|p_{\lambda}\right|\right\} \leq|z| \leq \max \left\{\left|c_{\lambda}\right|,\left|p_{\lambda}\right|\right\},
$$

and $\Gamma_{I}, \Gamma_{E}$, and $\Gamma_{\lambda}$ are symmetric with respect to rotations by $2 \pi / m$.

Proof. We first explain the idea of the construction and later on we compute explicitly these preimages of the critical value circle $V_{\lambda}$. We observe that $V_{\lambda}$ contains all the critical values of $F_{\lambda}$ except the critical value located at infinity. We recall that $\infty$ is a critical point with multiplicity $n-1$ and 0 is also a critical point with mutlitplicity $d-1$. Thus the set $\mathbb{C} \backslash V_{\lambda}$ has two connected components, the first one is a punctured annulus $\mathcal{A}$ between $V_{\lambda}$ and $\infty$ and, the second one, a round disk $\mathcal{D}$ bounded by $V_{\lambda}$ (see Figure 5).

We claim that the preimage of the punctured annulus $\mathcal{A}$ are formed by two annuli, $A_{\infty}$ and $A_{0}$, the first one punctured at $\infty$ and the second punctured at 0 . To see the claim, we notice that by the Riemann-Hurwitz formula the preimage of an annulus without critical values is always an annulus and the only preimages of $\infty$ by $F_{\lambda}$ are $\infty$ and 0 . Moreover, the boundaries of the punctured annulus $A_{\infty}$ are $\infty$ and a Jordan curve, denoted by $\Gamma_{E}$, and by construction $F_{\lambda}\left(\Gamma_{E}\right)=V_{\lambda}$ with degree $n$. In a similar way, the boundaries of the punctured annulus $A_{0}$ are 0 and a Jordan curve, denoted by $\Gamma_{I}$ such that $F_{\lambda}\left(\Gamma_{I}\right)=V_{\lambda}$ with degree $d$. Finally, since by the Riemann-Hurwitz formula the preimage of a disk without critical values is always a simply connected region and the map between these two simply connected regions is conformal, the preimage of the disk $\mathcal{D}$ consists of $m$ simply connected regions, denoted by $\Omega_{j}$ for $0 \leq j \leq m-1$, each one containing one of the $m$ prepoles and such that $F_{\lambda}$ maps each $\Omega_{j}$ conformally onto the disk $\mathcal{D}$. See Figure 5. 
Now we can compute explicitly the two curves $\Gamma_{E}$ and $\Gamma_{I}$ preimages of the critical value circle $V_{\lambda}$. For this, we write $v_{\lambda}=\mu \lambda^{n / m}$, so that $\mu=$ $m / d(d / n)^{n / m}$, see Equation (2.2). We search for the values of $z$ such that $\left|F_{\lambda}(z)\right|=\left|v_{\lambda}\right|$, since the radius of $V_{\lambda}$ is $\left|v_{\lambda}\right|$. We write $z=r|\lambda| \frac{1}{m} e^{i \varphi}$ with $r \geq 0$, and using the definition of $v_{\lambda}=F_{\lambda}\left(c_{\lambda}\right)$ (see, Equation (2.2)) we have that

$$
\left|F_{\lambda}(z)\right|=|\lambda|^{\frac{n}{m}} r^{n}\left|1+r^{-m} e^{i(\operatorname{Arg}(\lambda)-m \varphi)}\right|=\left|v_{\lambda}\right|=\mu|\lambda|^{n / m},
$$

where $\lambda=|\lambda| e^{i \operatorname{Arg}(\lambda)}$. We define the auxiliary function

$$
H(r, \varphi)=r^{2 n}\left|1+r^{-m} e^{i(\operatorname{Arg}(\lambda)-m \varphi)}\right|^{2}=r^{2 n}+2 r^{n-d} \cos (\operatorname{Arg}(\lambda)-m \varphi)+r^{-2 d},
$$

and now the preimage of $V_{\lambda}$ writes as $H(r, \varphi)=\mu^{2}$. We obviously have that

$$
q_{\text {min }}(r)=r^{2 n}-2 r^{n-d}+r^{-2 d} \leq H(r, \varphi) \leq r^{2 n}+2 r^{n-d}+r^{-2 d}=q_{\max }(r) .
$$

It is easy to see that $q_{\min }$ has minimum at $r=1$ with $q_{\min }(1)=0$ and $q_{\max }$ has a minimum at $r=(d / n)^{\frac{1}{m}}$ with $q_{\max }\left((d / n)^{\frac{1}{m}}\right)=\mu^{2}$. See Figure 4 . Computing the derivative of $H(r)$ with respect to $r$ gives

$$
\frac{\partial H}{\partial r}(r, \varphi)=2 r^{-2 d-1}\left(n r^{2 m}+(n-d) r^{m} \cos (\operatorname{Arg}(\lambda)-m \varphi)-d\right) .
$$

Now let $\rho=r^{m}$ and let $g(\rho)=n \rho^{2}+(n-d) \rho \cos (\operatorname{Arg}(\lambda)-m \varphi)-d$. We see that there exists a unique $\rho_{*}(\varphi)>0$ for which $g\left(\rho_{*}(\varphi)\right)=0$ given by,

$$
\frac{1}{2}\left(\frac{d-n}{n} \cos (\operatorname{Arg}(\lambda)-m \varphi)+\sqrt{\left(\frac{n-d}{n}\right)^{2} \cos ^{2}(\operatorname{Arg}(\lambda)-m \varphi)+4 d / n}\right)
$$

Then, for each $\varphi$ the function $H(r, \varphi)$ has a minimum at $r_{*}(\varphi)=\rho_{*}(\varphi)^{\frac{1}{m}}$. By (3.2) we see that for each $\varphi$,

$$
\lim _{r \rightarrow 0^{+}} H(r, \varphi)=\lim _{r \rightarrow \infty} H(r, \varphi)=\infty .
$$

Hence we conclude that $H\left(r_{*}, \varphi\right) \leq \mu^{2}$. See Figure 4. Then there exists three unique functions $r_{I}(\varphi), r_{*}(\varphi)$ and $r_{E}(\varphi)$, such that $r_{I}(\varphi) \leq r_{*}(\varphi) \leq r_{E}(\varphi)$ and $H\left(r_{I}(\varphi), \varphi\right)=H\left(r_{E}(\varphi), \varphi\right)=\mu^{2}$. Thus we can define 


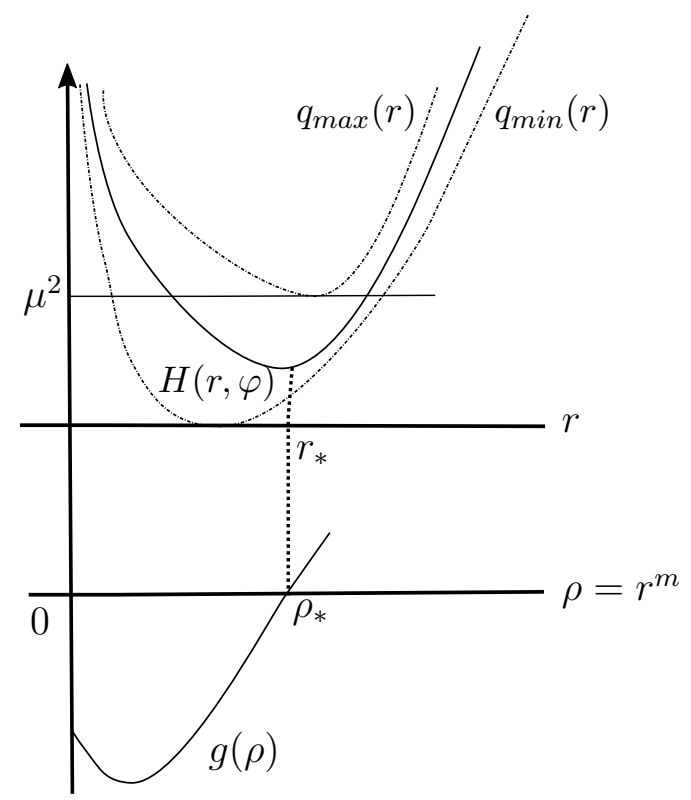

Figure 4: Diagram for the proof of Proposition 3.1, for fixed $\varphi$.

- $\Gamma_{E}(\varphi)=|\lambda|^{\frac{1}{m}} \cdot r_{E}(\varphi) \cdot e^{i \varphi}$

- $\Gamma_{I}(\varphi)=|\lambda|^{\frac{1}{m}} \cdot r_{I}(\varphi) \cdot e^{i \varphi}$

- $\Gamma_{\lambda}(\varphi)=|\lambda| \frac{1}{m} \cdot r_{*}(\varphi) \cdot e^{i \varphi}$

By construction we have that $F_{\lambda}\left(\Gamma_{E}\right)=F_{\lambda}\left(\Gamma_{I}\right)=V_{\lambda}$ and $F_{\lambda}\left(\Gamma_{\lambda}\right) \subset$ $\overline{\mathcal{D}}$. We now turn our attention to the curve $\Gamma_{\lambda}$. First, we observe that if $\operatorname{Arg}(\lambda)-m \varphi=0(\bmod 2 \pi)$, then $r_{I}(\varphi)=r_{E}(\varphi)=r_{*}(\varphi)=(d / n)^{\frac{1}{m}}$ and $\Gamma_{\lambda}\left(r_{E}(\varphi)\right)=\Gamma_{\lambda}\left(r_{I}(\varphi)\right)=\Gamma_{\lambda}\left(r_{*}(\varphi)\right)=(\lambda d / n)^{\frac{1}{m}}$, thus the three curves pass trough the critical points $c_{\lambda}$. If $\operatorname{Arg}(\lambda)-m \varphi \neq 0(\bmod 2 \pi)$, then $r_{I}<r_{*}<r_{E}$. Finally, if $\operatorname{Arg}(\lambda)-m \varphi=\pi(\bmod 2 \pi)$ then we obtain $r_{*}=1$ and $\Gamma_{\lambda}(\varphi)=(-\lambda)^{\frac{1}{m}}$, proving that $\Gamma_{\lambda}$ passes trough the prepoles $p_{\lambda}$.

The maximum and minimum of $\rho_{*}(\varphi)$, see Equation (3.3), are determined by the maximum and minimum of the term $\frac{d-n}{n} \cos (\operatorname{Arg}(\lambda)-m \varphi)$, that depends on the values of $d$ and $n$. When $n \geq d$ we see that the maximum is obtained for $\operatorname{Arg}(\lambda)-m \varphi=0(\bmod 2 \pi)$, and the minimum for $\operatorname{Arg}(\lambda)-$ $m \varphi=\pi(\bmod 2 \pi)$, while for $d>n$ the opposite happens. It follows that

$$
\min \left\{1,(d / n)^{\frac{1}{m}}\right\} \leq r_{*}(\varphi) \leq \max \left\{1,(d / n)^{\frac{1}{m}}\right\}
$$


Thus, if $z$ lies in $\Gamma_{\lambda}$ we see that $\min \left\{\left|c_{\lambda}\right|,\left|p_{\lambda}\right|\right\} \leq|z| \leq \max \left\{\left|c_{\lambda}\right|,\left|p_{\lambda}\right|\right\}$. Finally, by construction these three curves are symmetric with respect to rotations around the origin by $z \rightarrow \nu z$, with $\nu=e^{i 2 \pi / m}$.

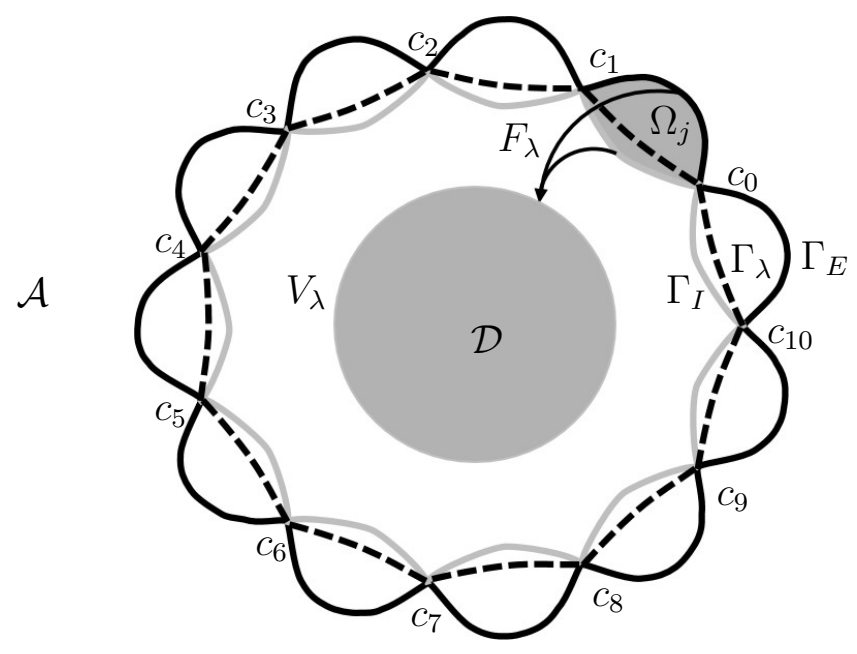

Figure 5: The curves $\Gamma_{I}$ (grey), $\Gamma_{E}$ (solid black) and $\Gamma_{\lambda}$ (dashed) when $n=5$, $d=6$ and then $m=11$. These three curves intersect at the critical points. The region $\Omega_{j}$ between $\Gamma_{I}$ and $\Gamma_{E}$ contains a prepole that lies in the curve $\Gamma_{\lambda}$, and it is mapped by $F_{\lambda}$ in a one-to-one fashion over $\mathcal{D}$. For $n=d \geq 3$ the curve $\Gamma_{\lambda}$ coincides with the critical circle.

Figure 5 illustrates the curves when $\lambda$ is small so that $\left|v_{\lambda}\right|<\min \left\{\left|c_{\lambda}\right|,\left|p_{\lambda}\right|\right\}$. If $n=d$ then critical points and prepoles are at the same distance from the origin. Instead, if $n>d$ we see that the critical points are closer to the origin than the prepoles, and if $d>n$ then the prepoles are closer to the origin than the critical points.

The image of $\Gamma_{\lambda}$ is a "flower" that passes successively through the critical values and the origin and it is contained in the closure of the critical value circle.

It follows from the previous proposition that there are $m$ domains $\Omega_{j}$ with $j=0,1, \ldots, m-1$, whose boundaries consist of $\Gamma_{I}$ and $\Gamma_{E}$ in between two consecutive critical points. Each of these domains $\Omega_{j}$ contains a prepole inside and is mapped in a one-to-one fashion onto $\mathcal{D}$.

The following result is a direct consequence of Proposition 3.1. 
Proposition 3.2 The curves $\Gamma_{I}$ and $\Gamma_{E}$ that are the preimages of $V_{\lambda}$ are such that

(a) $F_{\lambda}$ takes the curve $\Gamma_{E}$ passing through two consecutive critical points one-to-one onto a sector of $V_{\lambda}$ whose argument is $\frac{2 \pi n}{m} . F_{\lambda}$ takes the curve $\Gamma_{I}$ passing through two consecutive critical points one-to-one onto the remainder sector of $V_{\lambda}$ whose argument is $\frac{2 \pi d}{m}$.

(b) $F_{\lambda}$ maps the domain $\Omega_{j}$ that is enclosed by the curves $\Gamma_{I}$ and $\Gamma_{E}$ between two consecutive critical points in a one-to-one fashion onto the domain $\mathcal{D}$.

(c) $F_{\lambda}$ maps the curve $\Gamma_{E}$ in an n-to-one fashion, and the curve $\Gamma_{I}$ in a $d$-to-one fashion, both onto the critical value circle $V_{\lambda}$.

In the parameter $\lambda$-plane we restrict our attention to the region $\mathcal{O}$ given by

$$
\mathcal{O}=\left\{\lambda \in \mathbb{C}\left|\lambda_{\min }<\right| \lambda \mid<\lambda_{\max } \quad \text { and } \quad|\operatorname{Arg}(\lambda)|<\frac{\pi}{n-1}\right\} .
$$

See subsection 2.6 for the definition and properties of $\lambda_{\min }$ and $\lambda_{\max }$. For $\lambda \in \mathcal{O}$, we denote by $c_{0}=c_{0}(\lambda)$ the unique critical point with $\left|\operatorname{Arg}\left(c_{0}\right)\right|<$ $\pi / m$. We notice that if $\lambda \in \mathbb{R}^{+}$then $c_{0} \in \mathbb{R}^{+}$.

Hereafter we parametrize $\Gamma_{\lambda}(\theta)$ for $\theta \in \mathbb{R}$ and we recall that this curve joins critical points and prepoles in the counterclockwise direction. So $\Gamma_{\lambda}(\theta)$ is $2 \pi$-periodic in $\theta$ and depends analytically on $\lambda$ for $\lambda \in \mathcal{O}$. We fix $\Gamma_{\lambda}(0)=$ $\Gamma_{\lambda}(2 \pi)=c_{0}(\lambda)$.

In the next proposition we bound the position of the curve $\Gamma_{\lambda}$ when the parameter $\lambda$ belongs to $\mathcal{O}$, see Equation (2.6).

Proposition 3.3 The curve $\Gamma_{\lambda}$ is contained in the region $\left\{z \in \mathbb{C} ; v_{\lambda_{\min }} \leq\right.$ $\left.|z| \leq v_{\lambda_{\max }}\right\}$ for all $\lambda$-values in $\mathcal{O}$, where $v_{\lambda_{\min }}=(1 / 2)^{\frac{n d}{n d-m}}(d / m)^{\frac{m}{n d-m}}$ and $v_{\lambda_{\max }}=2$.

Proof. Let $\lambda$ be a parameter in $\mathcal{O}$ then we have that $c_{\lambda_{\min }} \leq\left|c_{\lambda}\right| \leq c_{\lambda_{\max }}$, $v_{\lambda_{\min }} \leq\left|c_{\lambda}\right| \leq v_{\lambda_{\max }}$ and $p_{\lambda_{\min }} \leq\left|p_{\lambda}\right| \leq p_{\lambda_{\max }}$, see Equations (2.1) and (2.3). Using the definition of $v_{\lambda}=F_{\lambda}\left(c_{\lambda}\right)$ we have that,

$$
\begin{aligned}
& v_{\lambda_{\min }}=F_{\lambda_{\min }}\left(c_{\lambda_{\min }}\right)=(1 / 2)^{\frac{n d}{n d-m}}(d / m)^{\frac{m}{n d-m}} \\
& v_{\lambda_{\max }}=F_{\lambda_{\max }}\left(c_{\lambda_{\max }}\right)=2
\end{aligned}
$$


We recall that for any parameter $\lambda$ the critical circle $C_{\lambda}$, the critical value circle $V_{\lambda}$ and the prepole circle $P_{\lambda}$ have radii $\left|c_{\lambda}\right|,\left|v_{\lambda}\right|$ and $\left|p_{\lambda}\right|$, respectively. Moreover, for all $z$ in the curve $\Gamma_{\lambda}$, see Proposition 3.1,

$$
\min \left\{\left|c_{\lambda}\right|,\left|p_{\lambda}\right|\right\} \leq|z| \leq \max \left\{\left|c_{\lambda}\right|,\left|p_{\lambda}\right|\right\}
$$

First we show that $v_{\lambda_{\max }}>\max \left\{c_{\lambda_{\max }}, p_{\lambda_{\max }}\right\}$. On the one hand, since $d / m<1$, we have that $c_{\lambda_{\max }}=\left(\frac{2 d}{m}\right)^{1 / n}<2=v_{\lambda_{\max }}$. On the other hand, we have $p_{\lambda_{\max }}=(n / d)^{1 / m} 2^{1 / n}(d / m)^{1 / n}$ and we need to analyze different cases. If $d \geq n$ then by the above inequality we conclude that $p_{\lambda_{\max }} \leq c_{\lambda_{\max }}<2$. If $n>d$ we see that

$$
p_{\lambda_{\text {max }}}=n^{1 / m} 2^{1 / n} d^{\frac{d}{n m}}\left(\frac{1}{m}\right)^{1 / n}<n^{1 / m} 2^{1 / n} n^{\frac{d}{n m}}\left(\frac{1}{m}\right)^{1 / n}=\left(\frac{n}{m}\right)^{1 / n} 2^{1 / n}<2 .
$$

Second we show that $v_{\lambda_{\min }}<\min \left\{c_{\lambda_{\min }}, p_{\lambda_{\min }}\right\}$. To do this we observe that

$c_{\lambda_{\min }}=\left(\frac{d}{2 m}\right)^{\frac{d}{n d-m}}=\left(\frac{1}{2}\right)^{\frac{d}{n d-m}}\left(\frac{d}{m}\right)^{\frac{d}{n d-m}}>\left(\frac{1}{2}\right)^{\frac{n d}{n d-m}}\left(\frac{d}{m}\right)^{\frac{m}{n d-m}}=v_{\lambda_{\min }}$.

For the inequality that corresponds to $p_{\lambda_{\min }}=(n / d)^{1 / m}(d / 2 m)^{d /(n d-m)}$, we compute $v_{\lambda_{\text {min }}} / p_{\lambda_{\text {min }}}$ and show it is smaller than 1 . We start with

$$
\frac{v_{\lambda_{\min }}}{p_{\lambda_{\min }}}=\left(\frac{1}{2}\right)^{\frac{d(n-1)}{n d-m}}\left(\frac{d}{n}\right)^{\frac{1}{m}}\left(\frac{d}{m}\right)^{\frac{n}{n d-m}}
$$

and notice that, since $d(n-1) /(n d-m)>1$ we have

$$
\frac{v_{\lambda_{\min }}}{p_{\lambda_{\min }}}<\frac{1}{2}\left(\frac{d}{n}\right)^{\frac{1}{m}}\left(\frac{d}{m}\right)^{\frac{n}{n d-m}} .
$$

Then, it is enough to show that

$$
\left(\frac{d}{n}\right)^{\frac{1}{m}}\left(\frac{d}{m}\right)^{\frac{n}{n d-m}}<2
$$

or, equivalently, that 


$$
\frac{d}{n}<2^{m}\left(\frac{m}{d}\right)^{\frac{m n}{n d-m}} .
$$

But this is clear, since

$$
\frac{d}{n}<2^{n+d}
$$

This last inequality follows easily from elementary calculus. Then $v_{\lambda_{\min }}<$ $p_{\lambda_{\min }}$, as we wanted to show.

Now, we are ready to prove the existence of the first ring $\mathcal{S}^{1}$ in the parameter plane. To do so, we transfer the curve $\Gamma_{\lambda}$ constructed in the dynamical plane to the parameter $\lambda$-plane, using the Schwarz Lemma.

Proposition 3.4 The ring $\mathcal{S}^{1}$ in parameter space is a simple closed curve surrounding the McMullen domain of $F_{\lambda}$, such that it passes through $n-1$ centers of Sierpinski holes with escpape time equal to 3, and $n-1$ superstable parameter values where a critical point of $F_{\lambda}$ is periodic of period $q$ for some $q \in \mathbb{N}$.

Proof. We consider the simply connected region defined in the parameter plane $\mathcal{O}$ given by,

$$
\mathcal{O}=\left\{\lambda \in \mathbb{C}\left|\lambda_{\min }<\right| \lambda \mid<\lambda_{\max } \quad \text { and } \quad|\operatorname{Arg}(\lambda)|<\frac{\pi}{n-1}\right\} .
$$

Given a parameter $\lambda \in \mathcal{O}$, we denote by $c_{0}(\lambda)$ the critical point given by

$$
c_{0}(\lambda)=\left(\frac{d|\lambda|}{n}\right)^{1 / m} e^{i \operatorname{Arg}(\lambda) / m} .
$$

We claim that $c_{0}(\lambda)$ is a holomorphic map of $\lambda$. To see the claim we notice that $c_{0}(\lambda)=\exp \left(\frac{1}{m} \log (d \lambda / n)\right)$, where $\log$ denotes the principal value of the logarithm map and since $n \geq 2$, then $|\operatorname{Arg}(\lambda)|<\pi /(n-1) \leq \pi$.

We define the map $G: \mathcal{O} \mapsto W$ defined by $G(\lambda)=v_{0}(\lambda)$, where $v_{0}(\lambda)$ is the critical value of $F_{\lambda}$ corresponding to the critical point $c_{0}(\lambda)$. This map takes $\mathcal{O}$ onto the set $W$. Both sets $\mathcal{O}$ and $W$ are simply connected. It is easy to compute the set $W$, the precise values of $v_{\lambda_{\min }}$ and $v_{\lambda_{\max }}$ are given by Equation (3.4). We have, 


$$
W=\left\{z \in \mathbb{C} ; v_{\lambda_{\min }}<|z|<v_{\lambda_{\max }} \text { and }|\operatorname{Arg}(z)|<\frac{n \pi}{m(n-1)}\right\} .
$$

Also, by Proposition 3.3 the map $\Gamma_{\lambda}: \mathcal{O} \mapsto W$ defined by $\lambda \mapsto \Gamma_{\lambda}(\theta)$ takes $\mathcal{O}$ inside $W$. This function is analytic on its domain, and its image is bounded from the origin.

We finally define, for each value of $\theta$, the new map $Q(\lambda)=G^{-1}\left(\Gamma_{\lambda}(\theta)\right)$. Then applying the Schwarz Lemma to the map $Q$ we obtain a fixed point inside $\mathcal{O}$ so that there exists a unique value of $\lambda$, that we denote $\lambda_{\theta}$, such that for each $\theta$ the point $v_{\lambda_{\theta}} \in \Gamma_{\lambda}$, i.e., $\Gamma_{\lambda_{\theta}}(\theta)=v_{0}\left(\lambda_{\theta}\right)$. The set of $\lambda_{\theta}$ 's for which this happens varies continuously with $\theta$ and defines a simple closed curve in the parameter plane that is symmetric under rotation by $2 \pi / m$ and such that the critical value is mapped to the curve $\Gamma_{\lambda}$. This curve is the first curve $\mathcal{S}^{1}$.
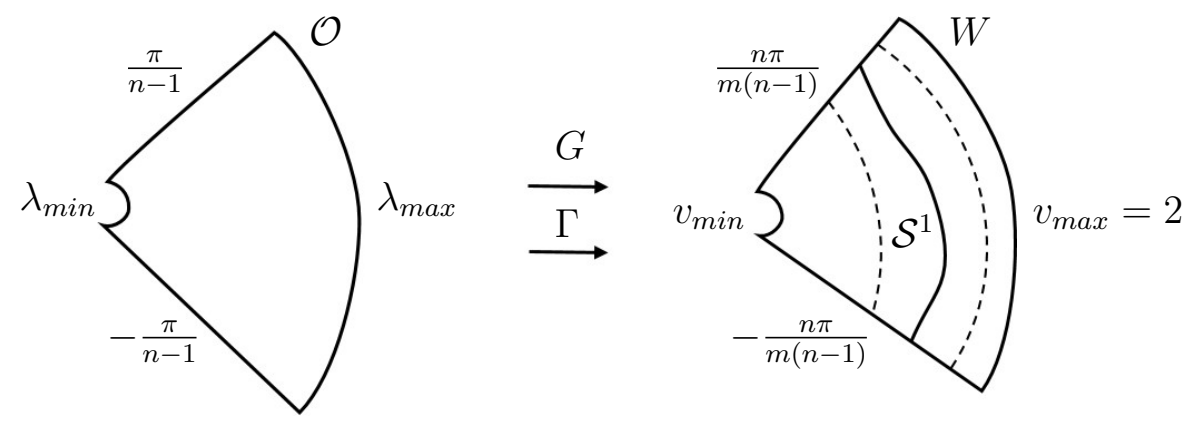

Figure 6: The mapping from set $\mathcal{O}$ to set $W$ in the parameter $\lambda$-plane.

For all points $z$ in $W$ we have that

$$
|\operatorname{Arg}(z)|<\frac{\pi}{m} \cdot \frac{n}{n-1}
$$

and this is larger than $2 \pi / m$. Then we see that in the region $\mathcal{O}$ we have exactly two parameters values $\lambda_{\theta_{1}}$ and $\lambda_{\theta_{2}}$, such that $v_{0}\left(\lambda_{\theta_{1}}\right)=c_{0}\left(\lambda_{\theta_{1}}\right)$ and $v_{0}\left(\lambda_{\theta_{2}}\right)=p_{0}\left(\lambda_{\theta_{2}}\right)$. The first one corresponding to the center of the main cardiod of the principal Mandelbrot set, and the second one corresponding to the center of a main Sierpiński hole. Then the critical value $v_{\lambda}$ coincides with one critical point and one prepole. Finally, using the symmetry of the 
parameter plane, see Theorem 2.2, we get $n-1$ cases where $\lambda_{\theta}$ corresponds to $v_{\lambda_{\theta}}=c_{\lambda_{\theta}}$ and $v_{\lambda_{\theta}}=p_{\lambda_{\theta}}$. These values locate the centers of the main cardioids of the $n-1$ principal Mandelbrot sets alternating the centers of $n-1$ Sierpinski holes.

Using the curve $\mathcal{S}^{1}$ we can divide the parameter $\lambda$-plane into two pieces bounded by $\mathcal{S}^{1}$. We denote by $I\left(\mathcal{S}^{1}\right)$ and $E\left(\mathcal{S}^{1}\right)$ the bounded and unbounded component of $\mathbb{C} \backslash \mathcal{S}^{1}$, respectively.

By Proposition 3.1 the image of $\Gamma_{\lambda}$ under $F_{\lambda}$ is contained in the closed disk bounded by the critical value circle $V_{\lambda}$ centered at the origin with radius $\left|v_{\lambda}\right|$. Therefore, we have the following result.

Proposition 3.5 The set $I\left(\mathcal{S}^{1}\right)$ contains all the parameters $\lambda$ such that the critical values $v_{\lambda}$ lie in the region bounded by $\Gamma_{\lambda}$. Moreover, when $\lambda \in I\left(\mathcal{S}^{1}\right)$ then $F_{\lambda}$ maps $\Gamma_{\lambda}$ strictly inside itself.

\subsection{Elementary Mapping Properties}

In the rest of the paper we restrict, for technical reasons, to the parameter values $\lambda$ in the following simply connected region depending on $d$ and $n$. Recall that if $n \leq d$ then $C_{\lambda} \succ P_{\lambda}$, and if $n>d$ then $P_{\lambda} \succ C_{\lambda}$, see $\S 2.2$.

- If $n \leq d$ then

$$
\mathcal{O}^{\prime}=\left\{\lambda \in \mathbb{C} ;|\lambda|>\lambda_{\text {min }}, \quad \lambda \in I\left(\mathcal{S}^{1}\right), \quad \text { and } \quad|\operatorname{Arg} \lambda|<\pi\right\} .
$$

- If $n>d$ then

$$
\mathcal{O}^{\prime}=\left\{\lambda \in \mathbb{C} ;|\lambda|>\lambda_{\text {min }}, \quad \lambda \in I\left(\mathcal{S}^{1}\right), \quad \text { and } \quad|\operatorname{Arg} \lambda|<\frac{d-1}{n-1} \pi\right\} .
$$

For example, in the case $n=5$ and $d=6$, as well as for all cases where $n=d$, we get that $|\operatorname{Arg} \lambda|<\pi$. Instead, for example, when $n=6, d=5$ we get $|\operatorname{Arg} \lambda|<4 \pi / 5$.

We recall that when $|\lambda|<\lambda_{\min }$, see Equation (2.6), then the Julia set of $F_{\lambda}$ is a Cantor set of circles. In addition, we observe that when $\lambda \in \mathcal{O}^{\prime}$ then $F_{\lambda}$ maps $\Gamma_{\lambda}$ strictly inside of itself, see Proposition 3.5.

As before, for $\lambda \in \mathcal{O}^{\prime}$, we denote by $c_{0}=c_{0}(\lambda)$ the unique critical point with $\left|\operatorname{Arg}\left(c_{0}\right)\right|<\pi / m$, where $c_{0} \in \mathbb{R}^{+}$if $\lambda \in \mathbb{R}^{+}$. 

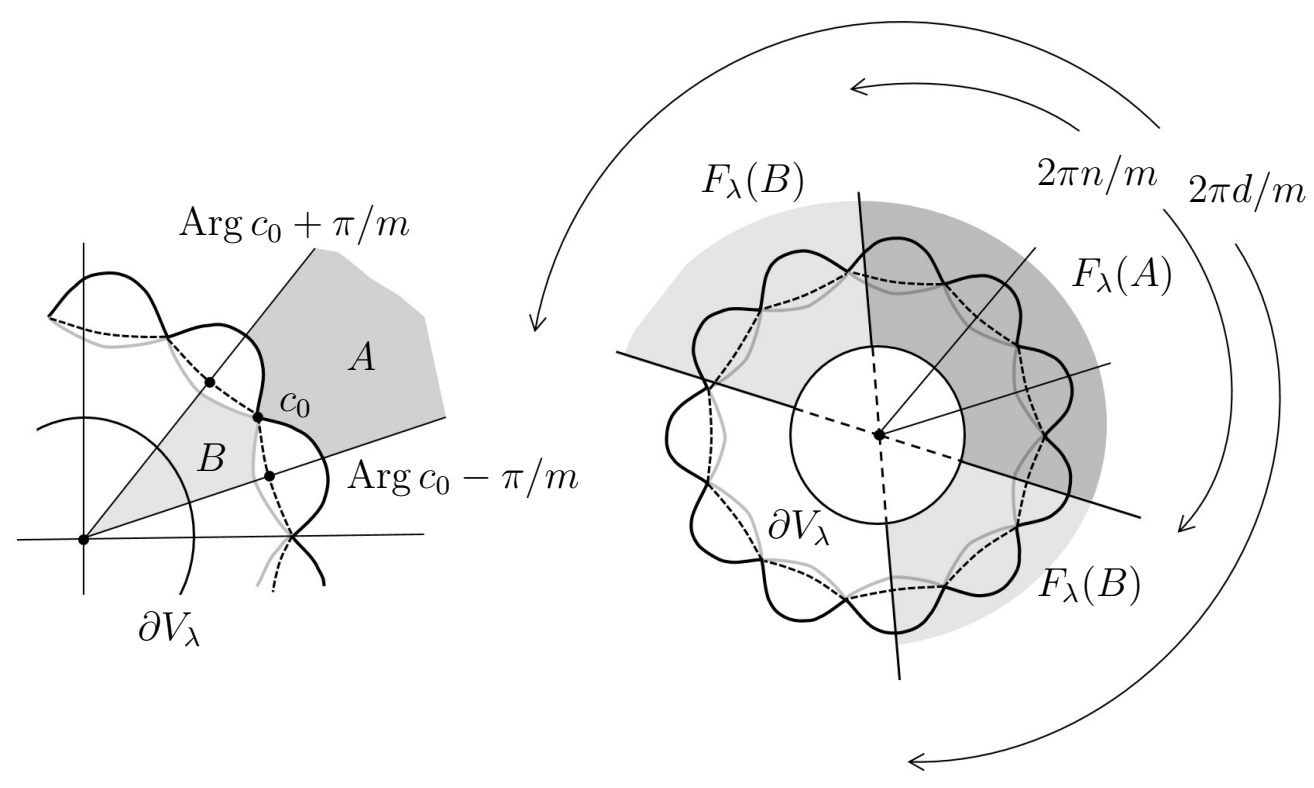

Figure 7: In this picture we illustrate the critical sectors $A, B$ and their images for the case $d>n(e . g ., n=5, d=6)$, and then $F_{\lambda}(A) \subset F_{\lambda}(B)$, with $|\lambda|$ small so that $\left|v_{\lambda}\right|<\left|c_{\lambda}\right|$. The critical point sector has argument $2 \pi / \mathrm{m}$. The image of $A$ (dark grey) lies in a sector with argument $2 \pi n / m$, and the image of $B$ (light grey) lies in a sector with argument $2 \pi d / m$.

Let $A$ be a sector bounded by two prepole rays corresponding to adjacent prepoles in the exterior of the curve $\Gamma_{E}$, and let $B$ be a sector bounded by two prepole rays corresponding to adjacent prepoles in the interior of the curve $\Gamma_{I}$, i.e., $A$ is an exterior sector, and $B$ is an interior sector in the dynamical plane with argument $2 \pi / \mathrm{m}$. We call $A$ a critical point exterior sector and $B$ a critical point interior sector since $A$ and $B$ contain at the "center" of their boundary a unique common critical point $c_{0}(\lambda)$ of $F_{\lambda}$. See Figure 7 .

We also define the following auxiliary sets,

$$
\begin{aligned}
& A_{-}=\left\{z \in A: \operatorname{Arg} c_{0}-\pi / m \leq \operatorname{Arg} z \leq \operatorname{Arg} c_{0}\right\}, \\
& B_{+}=\left\{z \in B ; \operatorname{Arg} c_{0} \leq \operatorname{Arg} z \leq \operatorname{Arg} c_{0}+\pi / m\right\} .
\end{aligned}
$$

It follows that,

Proposition 3.6 Let $F_{\lambda}(z)=z^{n}+\lambda / z^{d}$, with $1 / n+1 / d<1$. For $\lambda \in \mathcal{O}^{\prime}$ we have that, 


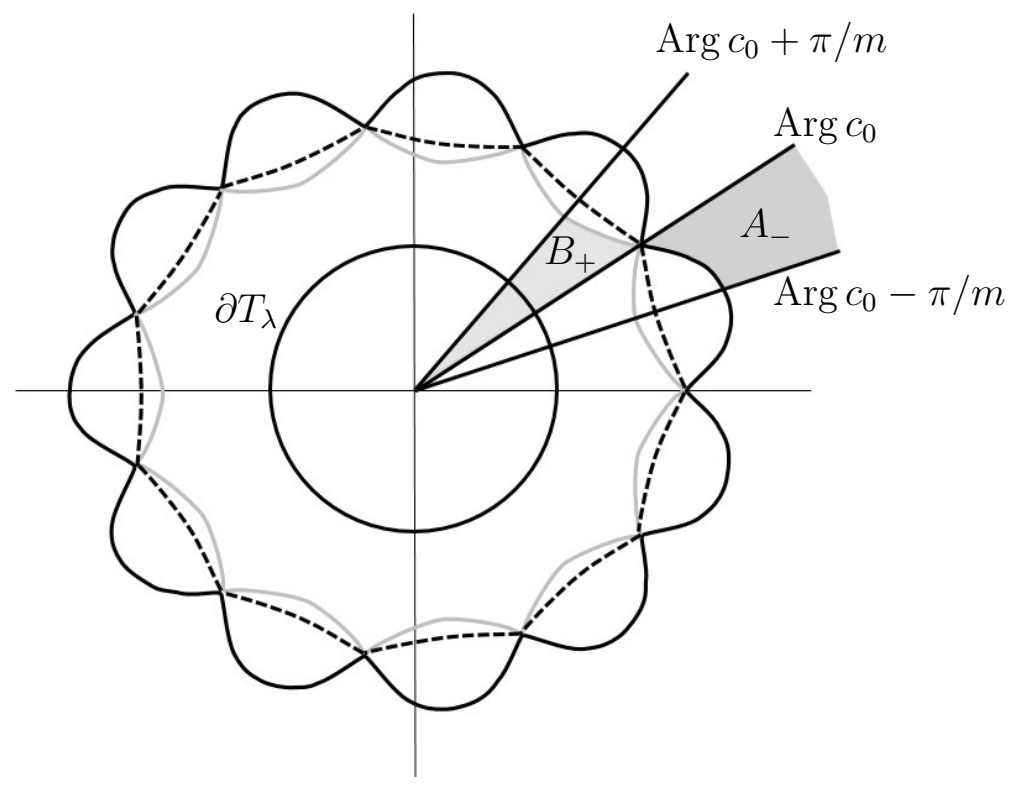

Figure 8: The regions $A_{-}$and $B_{+}$.

(a) $F_{\lambda}$ maps $A$ in a one-to-one fashion onto the sector with argument $\frac{2 \pi n}{m}$, bounded by the images of two prepole rays, i.e., two half straight lines in the exterior of the critical value circle $V_{\lambda}$.

(b) $F_{\lambda}$ maps $B$ in a one-to-one fashion onto the sector bounded by the opposite straight rays to the line boundaries of $F_{\lambda}(A)$ with argument $2 \pi d / m$, in the exterior of $V_{\lambda}$. The circular boundaries of $F_{\lambda}(A)$ and $F_{\lambda}(B)$ both contain $v_{\lambda}=F_{\lambda}\left(c_{\lambda}\right)$, the common boundary point of $A$ and $B$.

(c) Moreover, either $F_{\lambda}(A)$ or $F_{\lambda}(B)$ is contained in the other, that is, $A \subset F_{\lambda}(A) \subset F_{\lambda}(B)$ when $d \geq n$, and $A \subset F_{\lambda}(B) \subset F_{\lambda}(A)$ when $n>d$.

(d) If $\operatorname{Arg} \lambda>0$ then $A_{-} \subset F_{\lambda}\left(A_{-}\right) \cap F_{\lambda}\left(B_{+}\right)$.

Proof. The first two statements are clear from Proposition 3.2. We first prove the third one. Let $\lambda \in \mathcal{O}^{\prime}$. The straight line boundaries of $A$ and $B$ are the prepole rays and their arguments are denoted by $\operatorname{Arg} c_{0} \pm \pi / m$. Letting $z$ be a point of the images of the straight line boundaries, we have $\operatorname{Arg} z=n\left(\operatorname{Arg} c_{0} \pm \pi / m\right)$ or $\operatorname{Arg} z=d\left(\operatorname{Arg} c_{0} \mp \pi / m\right)$. See $\S 2.1$. 
Using the definition of the critical value $c_{0}$, we have that $\left|\operatorname{Arg}\left(c_{0}\right)\right|<\frac{\pi}{m}$ and we see that

$$
n\left(\operatorname{Arg} c_{0}-\frac{\pi}{m}\right)<\operatorname{Arg} c_{0}-\frac{\pi}{m}<0<\operatorname{Arg} c_{0}+\frac{\pi}{m}<n\left(\operatorname{Arg} c_{0}+\frac{\pi}{m}\right),
$$

and

$$
d\left(\operatorname{Arg} c_{0}-\frac{\pi}{m}\right)<\operatorname{Arg} c_{0}-\frac{\pi}{m}<0<\operatorname{Arg} c_{0}+\frac{\pi}{m}<d\left(\operatorname{Arg} c_{0}+\frac{\pi}{m}\right) .
$$

Since $2 \pi d / m+2 \pi n / m=2 \pi$ we see that, either $F_{\lambda}(A)$ or $F_{\lambda}(B)$ is contained in the other. If $d \geq n$ we obtain that $A \subset F_{\lambda}(A) \subset F_{\lambda}(B)$, and if $d<n$ we obtain the symmetric result $A \subset F_{\lambda}(B) \subset F_{\lambda}(A)$.

Now we prove the fourth statement. Let $\lambda \in \mathcal{O}^{\prime}$ with $\operatorname{Arg} \lambda>0$. The curve $\Gamma_{E}$ boundary of $A_{-}$lies outside the circular boundaries $V_{\lambda}$ of $F_{\lambda}\left(A_{-}\right)$ and $F_{\lambda}\left(B_{+}\right)$. On the one hand, we know that the arguments of the line boundaries of $A_{-}$are $\operatorname{Arg} c_{0}-\pi / m$ and $\operatorname{Arg} c_{0}$. In a similar way, the arguments of the line boundaries of $F_{\lambda}\left(A_{-}\right)$are $\operatorname{Arg} v_{0}=n \operatorname{Arg} c_{0}$ and $\operatorname{Arg} v_{0}-\pi n / m$. So we have that

$$
\operatorname{Arg} v_{0}-n \pi / m<\operatorname{Arg} c_{0}-\pi / m<0<\operatorname{Arg} c_{0}<\operatorname{Arg} v_{0}
$$

since $\operatorname{Arg} \lambda>0$. This shows that $A_{-} \subset F_{\lambda}\left(A_{-}\right)$.

On the other hand, the arguments of the line boundaries of $F_{\lambda}\left(B_{+}\right)$are $d \operatorname{Arg} c_{0}$ and $d \operatorname{Arg} c_{0}-d \pi / m$. Now using the different definition of $\mathcal{O}^{\prime}$ depending on $d$ and $n$, see Equations (3.5) and (3.6), we have that

- If $n \leq d$ then $\operatorname{Arg} c_{0}<\pi / m$ and $(n-1) \operatorname{Arg} c_{0}-\pi(d-1) / m<0$.

- If $n>d$ then

$$
\operatorname{Arg} c_{0}<\pi(d-1) /(m(n-1)) \text { and }(n-1) \operatorname{Arg} c_{0}-\pi(d-1) / m<0,
$$

Thus, since

$$
\operatorname{Arg} v_{0}-\frac{\pi d}{m}=\operatorname{Arg} c_{0}-\frac{\pi}{m}+(n-1) \operatorname{Arg} c_{0}-\frac{\pi(d-1)}{m},
$$

we get

$$
\operatorname{Arg} v_{0}-\frac{\pi d}{m}<\operatorname{Arg} c_{0}-\frac{\pi}{m}<0<\operatorname{Arg} c_{0}<\operatorname{Arg} v_{0} .
$$

Hence $A_{-} \subset F_{\lambda}\left(B_{+}\right)$, and the result follows. 


\subsection{Some Special Cases}

In this section we discuss the dynamics of several special cases of $F_{\lambda}$ that help define the rings around the McMullen domain.

First suppose that $\lambda>0$ and such that the critical point $c_{0}$ is a fixed point, see $\S 2.2$. If

$$
\lambda=\frac{n}{d}\left(\frac{d}{m}\right)^{\frac{m}{n-1}}>0,
$$

then

$$
v_{\lambda}=F_{\lambda}\left(c_{\lambda}\right)=\frac{m}{d}\left(\frac{d}{n}\right)^{\frac{n}{m}} \lambda^{\frac{n}{m}}=\left(\frac{d}{n} \lambda\right)^{\frac{1}{m}}=c_{\lambda},
$$

so that $c_{\lambda} \in \mathbb{R}^{+}$is a super-attracting fixed point. By the symmetry of the parameter plane, it follows that for $n-1$ other parameters $\omega^{j} \lambda$ with $j=0,1, \ldots, n-2$, where $\omega=e^{i 2 \pi /(n-1)}$, there is an iterate $F_{\lambda}^{q}$ for some $q \in \mathbb{N}$ with a super-attracting fixed point, see Theorem 2.2.

We next restrict attention to values of $\lambda$ lying in $\mathbb{R}^{+}$. Following [3], we can consider that there is a Mandelbrot set whose central spine lies along the interval $\left[\lambda_{-}, \lambda_{+}\right]$, where $\lambda_{-}$and $\lambda_{+}$correspond to the interval $[-2,1 / 4]$ of the actual Mandelbrot set, contained in $\mathbb{R}^{+}$. The existence of these and other copies of Mandelbrot sets in the parameter plane of $F_{\lambda}$ is given in [6, $20,21,22]$.

Proposition 3.7 (Superstable parameters for $\lambda \in \mathbb{R}^{+}$) There is a decreasing sequence of parameters in $\mathbb{R}^{+}$, such that $\lambda_{1}>\lambda_{2} \cdots$ converging to $\lambda_{-}$, and for $\lambda=\lambda_{k}$, the critical point $c_{0}$ is periodic with period $k$, and the critical orbit in $\mathbb{R}^{+}$has the special form when $k \in \mathbb{N}$ and $k \geq 2$ :

$$
0<v_{\lambda}=F_{\lambda}\left(c_{0}\right)<c_{0}=F_{\lambda}^{k}\left(c_{0}\right)<F_{\lambda}^{k-1}\left(c_{0}\right)<\cdots<F_{\lambda}^{3}\left(c_{0}\right)<F_{\lambda}^{2}\left(c_{0}\right) .
$$

In particular, $\lambda_{k}$ is a superstable parameter value of period $k$, and the orbit of $F_{\lambda_{k}}^{2}\left(c_{0}\right)$ is monotonically decreasing for $k-1$ iterations along $\mathbb{R}^{+}$.

We shall show below that each $\lambda_{k}$ lies on $\mathcal{S}^{k}$. Because of the $(n-1)$-fold symmetry in the parameter plane, we have a similar sequence of superstable parameter values along the ray $\lambda=\omega \cdot \mathbb{R}^{+}$, for $F_{\lambda}^{q}(z)$ and some $q \in \mathbb{N}$ with $\omega=e^{i \frac{2 \pi}{n-1}}$. 
Proposition 3.8 (Superstable parameters for $\lambda \in \omega \cdot \mathbb{R}^{+}$). Let $\lambda_{1}>\lambda_{2} \cdots$ be the decreasing sequence in $\mathbb{R}^{+}$defined in Proposition 3.7. Then there exists natural numbers $k^{\prime}, q$ and $r$ with $r \in\{0,1, \ldots, m-1\}$ such for $\lambda=\omega \lambda_{k}$ the orbit of the critical point $c_{r}$ is periodic with period $k q$ and the critical orbit along the line $\omega^{\frac{k^{\prime}}{q}} \cdot \mathbb{R}^{+}$has the special form when $k \geq 2$ :

$$
F_{\lambda}^{q}\left(c_{r}\right)<c_{r}=F_{\lambda}^{k q}\left(c_{r}\right)<F_{\lambda}^{(k-1) q}\left(c_{r}\right)<\cdots<F_{\lambda}^{3 q}\left(c_{r}\right)<F_{\lambda}^{2 q}\left(c_{r}\right) .
$$

Proof. Let $\nu=e^{i \frac{2 \pi}{m}}, \omega=e^{i \frac{2 \pi}{n-1}}$, and $\lambda=a \omega$ with $a \in \mathbb{R}^{+}$. Then $c_{0}=c_{\lambda}=$ $(d \omega a / n)^{\frac{1}{m}}$ and $c_{j}=c_{\lambda} \nu^{j}$, where $j=0,1, \ldots, m-1$. By Theorem 2.2, with $a$ in the role of $\lambda$, we get that there exist $k^{\prime}, q \in \mathbb{N}$ such that $F_{a \omega}^{q}\left(\omega^{\frac{k^{\prime}}{q}} z\right)=$ $\omega^{\frac{k^{\prime}}{q}} F_{a}^{q}(z)$. Then, for some $r, s \in \mathbb{N}$ we have that $k^{\prime} s=r(n-1)+1$ and $c_{r}=\nu^{r} c_{0}=\omega^{\frac{k^{\prime}}{q}}(d a / n)^{\frac{1}{m}} \in \omega^{\frac{k^{\prime}}{q}} \cdot \mathbb{R}^{+}$. Since $F_{\lambda}^{q}: \omega^{\frac{k^{\prime}}{q}} \cdot \mathbb{R}^{+} \rightarrow \omega^{\frac{k^{\prime}}{q}} \cdot \mathbb{R}^{+}$, the orbit of the critical point $c_{r}$ lies on $\omega^{\frac{k^{\prime}}{q}} \cdot \mathbb{R}^{+}$. For $a=\lambda_{k}$, then the critical point $c_{r}$ is periodic with period $k q$, the critical orbit for $F_{\lambda}^{q}$ along the line $\omega^{\frac{k^{\prime}}{q}} \cdot \mathbb{R}^{+}$ has the special abovementioned form when $k \geq 2$, as we wanted to show.

\subsection{Rings in Dynamical Plane}

In the rest of the paper we restrict, for technical reasons, to the parameter values $\lambda$ in the simply connected region $\mathcal{O}^{\prime}$ depending on $d$ and $n$, as defined in (3.5) and (3.6). Recall that when $\lambda \in \mathcal{O}^{\prime}$ then $F_{\lambda}$ maps $\Gamma_{\lambda}$ strictly inside itself, see Proposition 3.5.

For $\lambda \in \mathcal{O}^{\prime}$, we denote by $c_{0}=c_{0}(\lambda)$ the unique critical point with $\left|\operatorname{Arg}\left(c_{0}\right)\right|<\pi / m$. We notice that $c_{0} \in \mathbb{R}^{+}$if $\lambda \in \mathbb{R}^{+}$and thus the critical poins of $F_{\lambda}$ are located at $c_{j}=\nu^{j} c_{0}$ with $\nu=e^{i \frac{2 \pi}{m}}$ for $j=0,1, \ldots, m-1$.

In this section we prove the existence of infinitely many rings $\gamma_{\lambda}^{k}$ and $\xi_{\lambda}^{k}$ for $k=0,1,2, \ldots$ in the dynamical plane. Each ring $\gamma_{\lambda}^{k}$ (resp., $\xi_{\lambda}^{k}$ ) is a simple closed curve that is mapped $n^{k}$-to- 1 (resp., $d n^{k-1}$-to-1) onto the curve $\gamma_{\lambda}^{0}$ by $F_{\lambda}^{k}$. We shall use these rings in the next section to construct the rings $\mathcal{S}^{k}$ in the parameter plane, for $k>1$. We begin by defining $\gamma_{\lambda}^{0}$ to be the curve $\Gamma_{\lambda}$, defined in Proposition 3.1.

Let $E$ and $I$ be the exterior of $\Gamma_{E}$ and interior of $\Gamma_{I}$, respectively. If $\lambda \in \mathcal{O}^{\prime}$, then $F_{\lambda}$ maps $\gamma_{\lambda}^{0}$ strictly inside itself. By Proposition 3.2, it follows that $F_{\lambda}$ maps the exterior of the simple closed curve $\Gamma_{E}$ and the interior of 
$\Gamma_{I}$ as $n$-to- 1 and $d$-to- 1 covering, respectively, onto the exterior of the critical value circle $V_{\lambda}$. Therefore there are two preimages of $\gamma_{\lambda}^{0}$, that we denote by $\gamma_{\lambda}^{1}$ and $\xi_{\lambda}^{1}$ that lie in $E$ and $I$, respectively. The curve $\gamma_{\lambda}^{1}$ lies outside of $\gamma_{\lambda}^{0}$ and the curve $\xi_{\lambda}^{1}$ lies inside of $\gamma_{\lambda}^{0}$. The function $F_{\lambda}$ maps the curve $\gamma_{\lambda}^{1}$ (resp., $\left.\xi_{\lambda}^{1}\right)$ in an $n$-to-1 (resp., $d$-to-1) fashion onto $\gamma_{\lambda}^{0}$. See Figure 9.

Since $F_{\lambda}$ is a covering map in these regions, it follows that $\gamma_{\lambda}^{1}$ and $\xi_{\lambda}^{1}$ must be disjoint simple closed curves. Then $F_{\lambda}$ maps the exterior of $\gamma_{\lambda}^{1}, n$ to- 1 onto the exterior of $\gamma_{\lambda}^{0}$, and there is a preimage of $\gamma_{\lambda}^{1}$ lying in this region and mapped $n$-to- 1 onto $\gamma_{\lambda}^{1}$. Call this simple closed curve $\gamma_{\lambda}^{2}$.

Similarly, $F_{\lambda}$ maps the interior of $\xi_{\lambda}^{1}$ as a $d$-to- 1 covering onto the exterior of $\gamma_{\lambda}^{0}$, then there is a preimage of $\gamma_{\lambda}^{1}$ lying in the interior of $\xi_{\lambda}^{1}$ and mapped $d$-to-1 onto $\gamma_{\lambda}^{1}$. Call this simple closed curve $\xi_{\lambda}^{2}$.

Continuing inductively, we find a collection of simple closed curves $\gamma_{\lambda}^{k}$ and $\xi_{\lambda}^{k}$ for $k \geq 1$ having the properties that:

1. $\gamma_{\lambda}^{k+1}$ (resp., $\xi_{\lambda}^{k+1}$ ) lies in the exterior of $\gamma_{\lambda}^{k}$ (resp., interior of $\xi_{\lambda}^{k}$ );

2. $F_{\lambda}$ takes $\gamma_{\lambda}^{k+1}$ as an $n$-to- 1 covering onto $\gamma_{\lambda}^{k}$ and $F_{\lambda}$ takes $\xi_{\lambda}^{k+1}$ as a $d$-to-1 covering onto $\gamma_{\lambda}^{k}$;

3. so $F_{\lambda}^{k+1}$ takes $\gamma_{\lambda}^{k+1}$ as an $n^{k+1}$-to- 1 covering of $\gamma_{\lambda}^{0}$ and $F_{\lambda}^{k+1}$ takes $\xi_{\lambda}^{k+1}$ as a $d n^{k}$-to- 1 covering of $\gamma_{\lambda}^{0}$;

4. $\gamma_{\lambda}^{k+1}$ converge outward to the boundary of $B_{\lambda}$ as $k \rightarrow \infty$ and $\xi_{\lambda}^{k+1}$ converge inward to the boundary of $T_{\lambda}$ as $k \rightarrow \infty$.

We now construct a parametrization of each of the $\gamma_{\lambda}^{k}$ and $\xi_{\lambda}^{k}$. The curve $\gamma_{\lambda}^{0}$ is the curve $\Gamma_{\lambda}$ defined in Proposition 3.1. We parametrize it by $\gamma_{\lambda}^{0}(\theta)$ for $\theta \in \mathbb{R}$ and we recall that this curve joins critical points and prepoles in the counterclockwise direction. So $\gamma_{\lambda}^{0}(\theta)$ is $2 \pi$-periodic in $\theta$ and depends analytically on $\lambda$ for $\lambda \in \mathcal{O}^{\prime}$. We fix $\gamma_{\lambda}^{0}(0)=\gamma_{\lambda}^{0}(2 \pi)=c_{0}(\lambda)$. By construction of $\Gamma_{\lambda}$ (see Proposition 3.1) this curve is symmetric with respect to rotations around the origin by $z \rightarrow \nu z$, with $\nu=e^{i 2 \pi / m}$. So, we have that $\nu^{j} \gamma_{\lambda}^{0}(\theta)=$ $\gamma_{\lambda}^{0}(\theta+2 \pi j / m)$ for all $j$ and all $\theta$, in particular the rest of the critical points located at $\nu^{j} c_{0}$ correspond to $\gamma_{\lambda}^{0}(2 \pi j / m)$.

To parametrize $\gamma_{\lambda}^{k}$, consider the critical point exterior sector $A$ and the critical point interior sector $B$ in Proposition 3.6. We assume $A$ and $B$ contain the critical point $c_{0}(\lambda)$ in their boundaries, see Figure 7 . It follows that there is a unique point in $A$ mapped to $c_{0}$ by $F_{\lambda}$; call this point $\gamma_{\lambda}^{1}(0)$. 


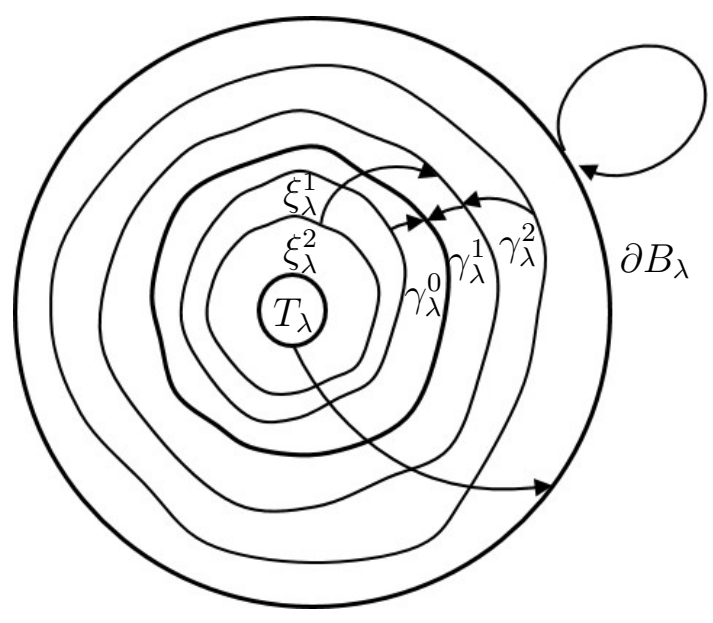

Figure 9: Schematic picture that shows the positions of the curves $\gamma_{\lambda}^{0}, \partial B_{\lambda}$ and $\partial T_{\lambda}$ (thick) and how they are mapped by $F_{\lambda}$. The curve $\gamma_{\lambda}^{0}$ is mapped inside of itself, the curves $\gamma_{\lambda}^{2}$, and $\xi_{\lambda}^{2}$ are mapped to $\gamma_{\lambda}^{1}$, and the curves $\gamma_{\lambda}^{1}$ and $\xi_{\lambda}^{1}$ are mapped to $\gamma_{\lambda}^{0}$.

There is also a unique point $\xi_{\lambda}^{1}(0) \in B$ mapped to $c_{0}$. Then we define $\gamma_{\lambda}^{1}(\theta)$ and $\xi_{\lambda}^{1}(\theta)$ by requiring that

$$
F_{\lambda}\left(\gamma_{\lambda}^{1}(\theta)\right)=F_{\lambda}\left(\xi_{\lambda}^{1}(\theta)\right)=\gamma_{\lambda}^{0}(\theta)
$$

and such that $\gamma_{\lambda}^{1}(\theta)$ and $\xi_{\lambda}^{1}(\theta)$ vary continuously with $\theta$. Note that $\gamma_{\lambda}^{1}(\theta)$ is $2 \pi n$-periodic and $\xi_{\lambda}^{1}$ is $2 \pi d$-periodic, respectively. The direction of $\xi_{\lambda}^{1}(\theta)$ is the opposite of $\gamma_{\lambda}^{1}(\theta)$. Since $\gamma_{\lambda}^{1}(0) \in A$ and $\xi_{\lambda}^{1}(0) \in B$, we proceed inductively to define $\gamma_{\lambda}^{k}(\theta)$ and $\xi_{\lambda}^{k}(\theta)$ such that $\gamma_{\lambda}^{k}(0) \in A$ and $\xi_{\lambda}^{k}(0) \in B$ are the unique points that are mapped by $F_{\lambda}$ to $\gamma_{\lambda}^{k-1}(0)$, and requiring that

$$
F_{\lambda}\left(\gamma_{\lambda}^{k}(\theta)\right)=F_{\lambda}\left(\xi_{\lambda}^{k}(\theta)\right)=\gamma_{\lambda}^{k-1}(\theta)
$$

For each $k, \gamma_{\lambda}^{k}(\theta)$ is $2 \pi n^{k}$ periodic and $\xi_{\lambda}^{k}(\theta)$ is $2 \pi d n^{k-1}$ periodic in $\theta$, and both depend analytically on $\lambda$. The following proposition shows the relationship between the parametrization and the symmetry.

Proposition 3.9 For each $k \in \mathbb{N}$ and $\theta \in \mathbb{R}, \nu^{j} \gamma_{\lambda}^{k}(\theta)=\gamma_{\lambda}^{k}\left(\theta+2 \pi j n^{k} / m\right)$ and $\nu^{j} \xi_{\lambda}^{k}(\theta)=\xi_{\lambda}^{k}\left(\theta-2 \pi j d n^{k-1} / m\right)$.

Proof. First when $k=1$, using Equations (2.4) and (3.7) we have 


$$
\begin{aligned}
F_{\lambda}\left(\nu^{j} \gamma_{\lambda}^{1}(\theta)\right)=\nu^{j n} F_{\lambda}\left(\gamma_{\lambda}^{1}(\theta)\right)=\nu^{j n} \gamma_{\lambda}^{0}(\theta)=\gamma_{\lambda}^{0}(\theta+2 \pi j n / m) \\
=F_{\lambda}\left(\gamma_{\lambda}^{1}(\theta+2 \pi j n / m)\right) .
\end{aligned}
$$

Then it follows that $\nu^{j} \gamma_{\lambda}^{1}(\theta)=\gamma_{\lambda}^{1}(\theta+2 \pi j n / m)$. For the induction argument, we assume that $\nu^{j} \gamma_{\lambda}^{k-1}(\theta)=\gamma_{\lambda}^{k-1}\left(\theta+2 \pi j n^{k-1} / m\right)$. Then, $\nu^{2 j} \gamma_{\lambda}^{k-1}(\theta)=$ $\nu^{j} \gamma_{\lambda}^{k-1}\left(\theta+2 \pi j n^{k-1} / m\right)=\gamma_{\lambda}^{k-1}\left(\theta+4 \pi j n^{k-1} / m\right)$, and

$$
\nu^{j n} \gamma_{\lambda}^{k-1}(\theta)=\gamma_{\lambda}^{k-1}\left(\theta+2 \pi j n^{k} / m\right) .
$$

Hence, using Equations (2.4) and (3.7), we have

$$
F_{\lambda}\left(\nu^{j} \gamma_{\lambda}^{k}(\theta)\right)=\nu^{j n} \gamma_{\lambda}^{k-1}(\theta)=\gamma_{\lambda}^{k-1}\left(\theta+2 \pi j n^{k} / m\right)=F_{\lambda}\left(\gamma_{\lambda}^{k}\left(\theta+2 \pi j n^{k} / m\right)\right) .
$$

We conclude that $\nu^{j} \gamma_{\lambda}^{k}(\theta)=\gamma_{\lambda}^{k}\left(\theta+2 \pi j n^{k} / m\right)$. Similarly, the result for $\xi_{\lambda}^{k}$ is proved.

In the next proposition we study the location of the $\operatorname{rings} \xi_{\lambda}^{k}(\theta)$ for certain values of $\theta$.

Proposition 3.10 If $\lambda \in \mathcal{O}^{\prime}$, then for each $k \geq 1$, if $n \leq d$ the portion of the ring $\xi_{\lambda}^{k}(\theta)$ with $|\theta| \leq 2 \pi d n^{k-1} / m$ lies in the region $|\operatorname{Arg} z|<4 \pi / m$, and if $n>d \geq 4$ it lies in the region $|\operatorname{Arg} z|<\pi \frac{n}{m}\left(\frac{d-1}{n-1}\right)$.

Proof. First we assume that $\lambda \in \mathcal{O}^{\prime}$ and $\operatorname{Arg} \lambda>0$. Since $c_{0} \in A_{-}$it follows, from Proposition 3.6, that $\xi_{\lambda}^{k}(0) \in B_{+}$. Then, since the arguments of the line boundaries of $B_{+} \operatorname{are} \operatorname{Arg} c_{0}$ and $\operatorname{Arg} c_{0}+\pi / m$, we obtain $\operatorname{Arg} c_{0}<$ $\operatorname{Arg} \xi_{\lambda}^{k}(0)<\operatorname{Arg} c_{0}+\pi / m$. See Figure 8 .

We deal first with the case $-2 \pi d n^{k-1} / m \leq \theta \leq 0$. The direction of $\xi_{\lambda}^{k}(\theta)$ is opposite to that of $\theta$, then $\operatorname{Arg} \xi_{\lambda}^{k}(0) \leq \operatorname{Arg} \xi_{\lambda}^{k}(\theta) \leq \operatorname{Arg} \xi_{\lambda}^{k}\left(-2 \pi d n^{k-1} / m\right)$. Since by Proposition 3.9, $\nu \xi_{\lambda}^{k}(0)=\xi_{\lambda}^{k}\left(-2 \pi d n^{k-1} / m\right)$, it follows that

$$
\begin{aligned}
0<\operatorname{Arg} c_{0} & <\operatorname{Arg} \xi_{\lambda}^{k}(0) \leq \operatorname{Arg} \xi_{\lambda}^{k}(\theta) \leq \operatorname{Arg} \xi_{\lambda}^{k}\left(-2 \pi d n^{k-1} / m\right)=\operatorname{Arg} \nu \xi_{\lambda}^{k}(0) \\
= & \frac{2 \pi}{m}+\operatorname{Arg} \xi_{\lambda}^{k}(0)<\frac{2 \pi}{m}+\operatorname{Arg} c_{0}+\frac{\pi}{m}=\frac{3 \pi}{m}+\frac{\operatorname{Arg} \lambda}{m}
\end{aligned}
$$

Using the different definition of $\mathcal{O}^{\prime}$ depending on $d$ and $n$, see Equations (3.5) and (3.6), we obtain that 
- For $n \leq d$, since $0<\operatorname{Arg} \lambda<\pi$, then $0<\operatorname{Arg} \xi_{\lambda}^{k}(\theta)<\frac{4 \pi}{m}$. Then we have that $\left|\operatorname{Arg} \xi_{\lambda}^{k}(\theta)\right|<4 \pi / m$.

- For $n>d \geq 4$, since $0<\operatorname{Arg} \lambda<\pi(d-1) /(n-1)$, then $0<\operatorname{Arg} \xi_{\lambda}^{k}(\theta)<$ $\frac{3 \pi}{m}+\frac{\pi}{m} \frac{d-1}{n-1} \leq \pi \frac{n}{m} \frac{d-1}{n-1}$. So, we conclude that $\left|\operatorname{Arg} \xi_{\lambda}^{k}(\theta)\right|<\pi \frac{n}{m} \frac{d-1}{n-1}$.

Now consider the case $0 \leq \theta \leq 2 \pi d n^{k-1} / m$. By Proposition 3.9, we have $\nu \xi_{\lambda}^{k}(\theta)=\xi_{\lambda}^{k}\left(\theta-2 \pi d n^{k-1} / m\right)=\xi_{\lambda}^{k}(t)$, where we let $t=\theta-2 \pi d n^{k-1} / m$ and $-2 \pi d n^{k-1} / m \leq t \leq 0$. It follows that $\xi_{\lambda}^{k}(\theta)=\nu^{-1} \xi_{\lambda}^{k}(t)$ and

$$
\operatorname{Arg} \xi_{\lambda}^{k}(\theta)=\operatorname{Arg} \xi_{\lambda}^{k}(t)-2 \pi / m
$$

Then, since $-2 \pi d n^{k-1} / m \leq t \leq 0$, it follows from Equation (3.9) that

$$
0<\operatorname{Arg} \xi_{\lambda}^{k}(t)<\frac{3 \pi}{m}+\frac{\operatorname{Arg} \lambda}{m} .
$$

Hence

$$
-\frac{2 \pi}{m}<\operatorname{Arg} \xi_{\lambda}^{k}(\theta)<\frac{\pi}{m}+\frac{\operatorname{Arg} \lambda}{m} .
$$

- For $n \leq d$, since $0<\operatorname{Arg} \lambda<\pi$, we have $-\frac{2 \pi}{m}<\operatorname{Arg} \xi_{\lambda}^{k}(\theta)<\frac{2 \pi}{m}$, and then it follows that $\left|\operatorname{Arg} \xi_{\lambda}^{k}(\theta)\right|<4 \pi / m$.

- For $n>d \geq 4$, since $0<\operatorname{Arg} \lambda<\pi \frac{d-1}{n-1}$, we have $-\frac{2 \pi}{m}<\operatorname{Arg} \xi_{\lambda}^{k}(\theta)<$ $\frac{\pi}{m}+\frac{\pi}{m} \frac{d-1}{n-1} \leq \pi \frac{n}{m} \frac{d-1}{n-1}$. It is easy to see that $\frac{2 \pi}{m}<\pi \frac{n}{m} \frac{d-1}{n-1}$, so we conclude that $\left|\operatorname{Arg} \xi_{\lambda}^{k}(\theta)\right| \leq \pi \frac{n}{m} \frac{d-1}{n-1}$.

Finally, since $\frac{3 \pi}{m}<\frac{(3 n-2) \pi}{m(n-1)}$, we have $\left|\operatorname{Arg} \xi_{\lambda}^{k}(\theta)\right|<\frac{(3 n-2) \pi}{m(n-1)}$. If $\operatorname{Arg} \lambda<0$, we invoke the $z \rightarrow \bar{z}$ symmetry in the parameter plane. Since $F_{\lambda}$ is conjugate to $F_{\bar{\lambda}}$ via $z \rightarrow \bar{z}$, it follows that the curves $\xi_{\lambda}^{k}(\theta)$ are mapped to $\xi_{\lambda}^{k}(-\theta)$ by the conjugacy. Hence these curves lie in the same region when $\operatorname{Arg} \lambda<0$. This concludes the proof.

We have shown the existence of the curves $\xi_{\lambda}^{k}(\theta)$, we have studied their parametrizations and positions. In the next section we translate these curves to the parameter plane to obtain the curves $\mathcal{S}^{k}$ to obtain our main result. 


\subsection{Rings in Parameter Plane}

Before turning to the proof of the existence of the rings in the parameter plane, we need to examine more carefully the parametrizations of the rings in the dynamical plane in two of the special cases discusses earlier, namely when $\lambda \in \mathbb{R}^{+}$and $\lambda \in \omega \cdot \mathbb{R}^{+}$, where $\omega=e^{i \frac{2 \pi}{n-1}}$.

First suppose that $\lambda \in \mathbb{R}^{+}$. For the special parameters $\lambda_{k}$ among the superstable parameters in $\mathbb{R}^{+}$, we have seen that $F_{\lambda_{k}}\left(c_{0}\right)$ always lies in $\mathbb{R}^{+}$ and satisfies (see Proposition 3.7),

$$
0<F_{\lambda_{k}}\left(c_{0}\right)<c_{0}=F_{\lambda_{k}}^{k}\left(c_{0}\right)<F_{\lambda_{k}}^{k-1}\left(c_{0}\right)<\cdots<F_{\lambda_{k}}^{3}\left(c_{0}\right)<F_{\lambda_{k}}^{2}\left(c_{0}\right) .
$$

Hence, $F_{\lambda_{k}}^{2}\left(c_{0}\right)$ lies on $\gamma_{\lambda_{k}}^{k-2} \cap \mathbb{R}^{+}$, and $F_{\lambda_{k}}^{j}\left(c_{0}\right)$ lies on $\gamma_{\lambda_{k}}^{k-j} \cap \mathbb{R}^{+}$, for $j=2, \ldots, k$. In particular, since the definition of the parametrization requires that

$$
F_{\lambda}\left(\gamma_{\lambda}^{j}(\theta)\right)=F_{\lambda}\left(\xi_{\lambda}^{j}(\theta)\right)=\gamma_{\lambda}^{j-1}(\theta)
$$

it follows that, for the special parameter value $\lambda_{k}$, we have

$$
\begin{aligned}
\gamma_{\lambda_{k}}^{0}(0) & =c_{0}, \\
\xi_{\lambda_{k}}^{k-1}(0) & =F_{\lambda_{k}}\left(c_{0}\right), \\
\gamma_{\lambda_{k}}^{k-2}(0) & =F_{\lambda_{k}}^{2}\left(c_{0}\right), \\
\gamma_{\lambda_{k}}^{k-3}(0) & =F_{\lambda_{k}}^{3}\left(c_{0}\right), \\
& \vdots \\
\gamma_{\lambda_{k}}^{1}(0) & =F_{\lambda_{k}}^{k-1}\left(c_{0}\right), \\
F_{\lambda_{k}}\left(\gamma_{\lambda_{k}}^{1}(0)\right) & =F_{\lambda_{k}}^{k}\left(c_{0}\right)=c_{0} .
\end{aligned}
$$

Next we turn our attention to the special parameter value $\omega \lambda_{k}$ lying along the line $\omega \cdot \mathbb{R}^{+}$in the parameter plane. For this we first need a result for $\lambda=\omega a$, with $a \in \mathbb{R}^{+}$.

We recall that the critical point ray passing through the critical point $c_{j}$ is denoted by $C_{j}$ and it is defined by the set of points $t c_{j}$ with $0<t<\infty$. See $\S 2.1$. If $v_{j}=F_{\lambda}\left(c_{j}\right)$ denotes the corresponding critical value, then the 
critical point ray $C_{j}$ is mapped under $F_{\lambda}$ in a two-to-one fashion onto a ray connecting $v_{j}$ to $\infty$.

We also introduce the following notation, if the critical point ray $C_{j_{1}}$ is mapped under $F_{\lambda}$ to the critical point ray $C_{j_{2}}$ and the ray $C_{j_{2}}$ is rotated by an angle $\alpha$ from ray $C_{j_{1}}$ in the counterclockwise direction, we write $C_{j_{1}} \stackrel{\alpha}{\longrightarrow} C_{j_{2}}$.

Proposition 3.11 Let $\lambda=\omega a$ with $a \in \mathbb{R}^{+}$, where $w=e^{i \frac{2 \pi}{n-1}}$. Then the following conditions hold,

(a) $F_{\lambda}$ maps critical point rays onto critical point rays, and if $C_{j}$ is a critical point ray then,

$$
C_{j} \stackrel{\frac{2 \pi}{m}(j(n-1)+1)}{\longrightarrow} C_{j(n-1)+1} .
$$

It follows then that critical point rays are eventually periodic under $F_{\lambda}$.

(b) For each $l \in \mathbb{N}, F_{\lambda}^{l}$ maps the critical point ray $C_{0}$ onto the critical point ray $C_{1+n+\ldots+n^{l-1}}$, and the rotation angle from ray $C_{0}$ to ray $C_{1+n+\ldots+n^{l-1}}$ is $2 \pi\left(1+n+\cdots+n^{l-1}\right) / m$. The next diagram follows,

$$
\begin{aligned}
& C_{0} \stackrel{\frac{2 \pi}{m}}{\longrightarrow} C_{1} \stackrel{\frac{2 \pi n}{m}}{\longrightarrow} C_{n+1} \stackrel{\frac{2 \pi n^{2}}{m}}{\longrightarrow} \cdots \\
& \stackrel{\frac{2 \pi n^{l-1}}{m}}{\longrightarrow} C_{1+n+\ldots+n^{l-1}} \stackrel{\frac{2 \pi n^{l}}{m}}{\longrightarrow} C_{1+n+\ldots+n^{l}} \cdots .
\end{aligned}
$$

Notice that in part $(a)$ the points in the rays may not be periodic, just the rays themselves are.

Proof. Let $t c_{j}$ with $0<t<\infty$ be the critical point ray passing through the critical point $c_{j}$ where $c_{j}=\nu^{j} c_{0}=\nu^{j}(d \lambda / n)^{1 / m}$ with $\nu=e^{i \frac{2 \pi}{m}}$. Its image is given by

$$
F_{\lambda}\left(t c_{j}\right)=\left(t^{n}+n / d t^{-d}\right) c_{j}^{n}=\left(t^{n}+n / d t^{-d}\right) c_{j} c_{j}^{n-1}
$$

with $c_{j}^{n-1}=\left(\nu^{j} c_{0}\right)^{n-1}=\left(\nu^{j}(d \omega a / n)^{1 / m}\right)^{n-1}$. Then we get

$$
F_{\lambda}\left(t c_{j}\right)=t^{\prime} c_{j n+1}
$$

where $t^{\prime}=\left(t^{n}+n / d t^{-d}\right)(d / n a)^{\frac{n-1}{m}}$, where we have used the fact that $\omega^{\frac{n-1}{m}}=$ $\nu$. Hence the critical point ray $C_{j}$ is mapped by $F_{\lambda}$ onto the critical point ray $C_{j n+1}$. Moreover, the angle from the ray $C_{j}$ to ray the $C_{j n+1}$ is $\operatorname{Arg} \nu^{j(n-1)+1}=$ 
$2 \pi(j(n-1)+1) / m$. Since the number of critical point rays is finite then they are eventually periodic by $F_{\lambda}$, proving the first statement.

For the second statement we proceed by induction. If $j=0$ then $C_{0}$ is mapped onto $C_{1}$ by $F_{\lambda}$ and the angle from ray $C_{0}$ to ray $C_{1}$ is $2 \pi / m$. So,

$$
C_{0} \stackrel{\frac{2 \pi}{m}}{\longrightarrow} C_{1}
$$

We assume that $F_{\lambda}^{l-1}$ maps the critical point ray $C_{0}$ onto the critical point ray $C_{1+n+\cdots+n^{l-2}}$ and then, using the first statement of this proposition and the fact that

$$
\left(1+n+\cdots+n^{l-2}\right) n+1=1+n+\cdots+n^{l-1},
$$

then the image of the ray $C_{1+n+\cdots+n^{l-2}}$ is the critical point ray $C_{1+n+\cdots+n^{l-1}}$.

Then $F_{\lambda}^{l}$ maps ray $C_{0}$ onto ray $C_{1+n+\cdots+n^{l-1}}$ and the rotation angle from ray $C_{1+n+\cdots+n^{l-2}}$ to ray $C_{1+n+\cdots+n^{l-1}}$ is $2 \pi n^{l-1} / m$. We can see that for each $l \in \mathbb{N}$ the critical point ray $C_{0}$ is mapped by $F_{\lambda}^{l}$ onto the critical point ray $C_{1+n+\ldots+n^{l-1}}$ with rotation angle $2 \pi\left(1+n+\ldots+n^{l-1}\right) / m$, as we wanted to show.

In $\S 3.4$ we showed the existence of infinitely many rings $\gamma_{\lambda}^{k}$ and $\xi_{\lambda}^{k}$ for $k=0,1,2, \ldots$ in the dynamical plane. Each ring $\gamma_{\lambda}^{k}$ is a simple closed curve such that $F_{\lambda}^{k}: \gamma_{\lambda}^{k} \mapsto \gamma_{\lambda}^{0}$ with degree $n^{k}$-to- 1 , and in a similar way $F_{\lambda}^{k}: \xi_{\lambda}^{k} \mapsto \gamma_{\lambda}^{0}$ with degree $d n^{k-1}$-to-1, where $\gamma_{\lambda}^{0}$ is the curve $\Gamma_{\lambda}$ defined in Proposition 3.1.

Thus, for $k=0,1,2, \ldots$ the point $F_{\lambda}^{k}\left(\gamma_{\lambda}^{k}\left(2 \pi\left(1+n+\cdots+n^{k-1}\right) / m\right)\right)=$ $\gamma_{\lambda}^{0}\left(2 \pi\left(1+n+\cdots+n^{k-1}\right) / m\right)$ lies on the critical point ray $C_{1+n+\ldots+n^{k-1}}$. It follows that the point $\gamma_{\lambda}^{k}\left(2 \pi\left(1+n+\cdots+n^{k-1}\right) / m\right)$ lies on the critical point ray $C_{0}$ and then, the point $F_{\lambda}\left(\gamma_{\lambda}^{k}\left(2 \pi\left(1+n+\cdots+n^{k-1}\right) / m\right)\right)=\gamma_{\lambda}^{k-1}(2 \pi(1+$ $\left.n+\cdots+n^{k-1}\right) / m$ ) lies on the critical point ray $C_{1}$.

On the other hand, by Proposition 3.9, for all $\theta$ we have $\nu \gamma_{\lambda}^{k}(\theta)=\gamma_{\lambda}^{k}(\theta+$ $\left.2 \pi n^{k} / m\right)$, setting $\beta_{k}=2 \pi\left(1+n+\cdots+n^{k-1}-n^{k}\right) / m$, we have $\gamma_{\lambda}^{k}\left(\beta_{k}\right)=$ $\nu^{-1} \gamma_{\lambda}^{k}\left(\beta_{k}+2 \pi n^{k} / m\right)$. It follows that the point $\gamma_{\lambda}^{k}\left(\beta_{k}\right)$ lies on the critical point ray $C_{-1}$. Since $F_{\lambda}^{k}\left(\xi_{\lambda}^{k}\left(\beta_{k}+2 \pi n^{k} / m\right)\right)=\gamma_{\lambda}^{0}\left(\beta_{k}+2 \pi n^{k} / m\right)$, it follows that the point $\xi_{\lambda}^{k}\left(\beta_{k}+2 \pi n^{k} / m\right)$ lies on the critical point ray $C_{0}$. See Figure 10 .

By the same result, for all $\theta$ we have $\nu \xi_{\lambda}^{l}(\theta)=\xi_{\lambda}^{k}\left(\theta-2 \pi d n^{k-1} / m\right)$, so $\xi_{\lambda}^{k}\left(\beta_{k}+2 \pi n^{k} / m-2 \pi d n^{k-1} / m\right)=\nu \xi_{\lambda}^{k}\left(\beta_{k}+2 \pi n^{k} / m\right)$. Hence the point $\xi_{\lambda}^{k}\left(\beta_{k}+\right.$ $\left.2 \pi n^{k} / m-2 \pi d n^{k-1} / m\right)$ lies on the critical point ray $C_{1}$. Similarly, the point $\xi_{\lambda}^{k}\left(\beta_{k}+2 \pi n^{k} / m+2 \pi d n^{k-1} / m\right)$ lies on the critical point ray $C_{-1}$. 


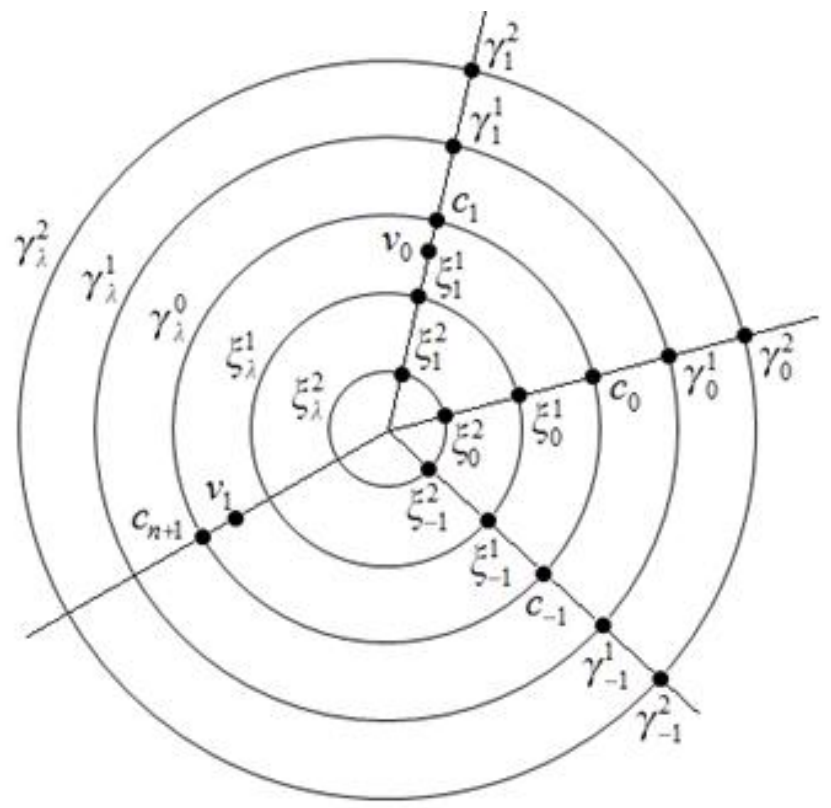

Figure 10: Parametrization of $\gamma_{\lambda}(\theta)$ when $\lambda=\omega a, a \in \mathbb{R}^{+}$, where $\gamma_{1}^{2}=$ $\gamma_{\lambda}^{2}\left(2 \pi\left(1+n+n^{2}\right) / m\right), \gamma_{0}^{2}=\gamma_{\lambda}^{2}(2 \pi(1+n) / m), \gamma_{-1}^{2}=\gamma_{\lambda}^{2}\left(2 \pi\left(1+n-n^{2}\right) / m\right)$. $\gamma_{1}^{1}=\gamma_{\lambda}^{1}(2 \pi(1+n) / m), \gamma_{0}^{1}=\gamma_{\lambda}^{1}(2 \pi / m)$, and $\gamma_{-1}^{1}=\gamma_{\lambda}^{1}(2 \pi(1-n) / m)$. Also, $\xi_{1}^{1}=\xi_{\lambda}^{1}(2 \pi(1-d) / m), \xi_{0}^{1}=\xi_{\lambda}^{1}(2 \pi / m)$, and $\xi_{-1}^{1}=\xi_{\lambda}^{1}(2 \pi(1+d) / m)$. Finally, $\xi_{1}^{2}=\xi_{\lambda}^{2}(2 \pi(1+n-n d) / m), \xi_{0}^{2}=\xi_{\lambda}^{2}(2 \pi(1+n) / m)$, and $\xi_{-1}^{2}=\xi_{\lambda}^{2}(2 \pi(1+n+$ $n d) / m)$.

We now turn to the proof of the existence of the rings $\mathcal{S}^{k}$ in the parameter plane for $k>1$. For technical reasons, we consider only the case when $n, d \geq$ 5. Set $a=\lambda_{k+1}$. It follows from Proposition 3.8 that, along the line $\omega^{\frac{k^{\prime}}{q}} \cdot \mathbb{R}^{+}$, for some $k^{\prime}, q \in \mathbb{N}$ there is a critical point $c_{r}$ for some $r \in\{0,1, \ldots, m-1\}$ such that its orbit has the special form when $k \geq 2$ :

$$
F_{\lambda}^{q}\left(c_{r}\right)<c_{r}=F_{\lambda}^{k q}\left(c_{r}\right)<F_{\lambda}^{(k-1) q}\left(c_{r}\right)<\cdots<F_{\lambda}^{3 q}\left(c_{r}\right)<F_{\lambda}^{2 q}\left(c_{r}\right) .
$$

Since $c_{r} \in \gamma_{\lambda}^{0}$, by Equation (3.7), we have that the set of curves given by $\left\{\gamma_{\lambda}^{0}, \xi_{\lambda}^{k}, \gamma_{\lambda}^{k-1}, \gamma_{\lambda}^{k-2}, \ldots, \gamma_{\lambda}^{1}\right\}$ is $k+1$ periodic. Then, $F_{\lambda}^{q}$ maps the curves in the following way, $\gamma_{\lambda}^{0} \rightarrow \xi_{\lambda}^{k} \rightarrow \gamma_{\lambda}^{k-1} \rightarrow \gamma_{\lambda}^{k-2} \rightarrow \cdots \rightarrow \gamma_{\lambda}^{1} \rightarrow \gamma_{\lambda}^{0}$.

Since $c_{0} \in \gamma_{\lambda}^{0}$ and $F_{\lambda}^{q}\left(c_{0}\right) \in \xi_{\lambda}^{k}$, then by Proposition 3.11, $F_{\lambda}^{q}\left(c_{0}\right) \in C_{1}$, 
that is,

$$
F_{\lambda}^{q}\left(c_{0}\right) \in \xi_{\lambda}^{k} \cap C_{1}
$$

To simplify the notation we define,

$$
\alpha_{k}=2 \pi\left(1+2+\cdots+n^{k-1}-d n^{k-1}\right) / m .
$$

The point $\xi_{\lambda}^{k}\left(\alpha_{k}\right)$ lies on the critical point ray $C_{1}$, and then $v_{\lambda}=v_{0}=$ $F_{\lambda}\left(c_{0}\right)=\xi_{\lambda}^{k}\left(\alpha_{k}\right)$.

Theorem 3.12 Let $n, d \geq 5$. For each $k \geq 1$ and every $\theta$ satisfying $|\theta| \leq$ $2 \pi d n^{k-1} / m$, there exists a unique parameter $\lambda=\lambda_{\theta, k}$ such that $v_{\lambda}=F_{\lambda}\left(c_{0}\right)=$ $\xi_{\lambda}^{k}(\theta)$.

Proof. We consider the simply connected region defined in the parameter plane $\mathcal{O}^{\prime}$ depending on the values of $n$ and $d$ given by,

- If $n \leq d$ then $\mathcal{O}^{\prime}=\left\{\lambda \in \mathbb{C} ;|\lambda|>\lambda_{\text {min }}, \lambda \in I\left(\mathcal{S}^{1}\right)\right.$, and $\left.\quad|\operatorname{Arg} \lambda|<\pi\right\}$.

- If $n>d$ then

$$
\mathcal{O}^{\prime}=\left\{\lambda \in \mathbb{C} ;|\lambda|>\lambda_{\text {min }}, \lambda \in I\left(\mathcal{S}^{1}\right), \text { and } \quad|\operatorname{Arg} \lambda|<\frac{d-1}{n-1} \pi\right\} .
$$

Given a parameter $\lambda \in \mathcal{O}^{\prime}$, we denote by $c_{0}(\lambda)$ the critical point given by

$$
c_{0}(\lambda)=\left(\frac{d|\lambda|}{n}\right)^{1 / m} e^{i \operatorname{Arg}(\lambda) / m} .
$$

We claim that $c_{0}(\lambda)$ is a holomorphic map of $\lambda$. To see this, we notice that $c_{0}(\lambda)=\exp \left(\frac{1}{m} \log (d \lambda / n)\right)$, where $\log$ denotes the principal value of the logarithm map. We observe that, in both cases, the critical point $c_{0}$ is such that $\left|\operatorname{Arg} c_{0}\right|<\pi / m$.

We define the map $G: \mathcal{O}^{\prime} \mapsto W^{\prime}$ defined by $G(\lambda)=v_{0}(\lambda)$, where $v_{0}(\lambda)$ is the critical value of $F_{\lambda}$ corresponding to the critical point $c_{0}(\lambda)$. This map takes $\mathcal{O}^{\prime}$ onto the set $W^{\prime}$. Both sets $\mathcal{O}^{\prime}$ and $W^{\prime}$ are simply connected. The set $W^{\prime}$ is given in the following way,

- If $n \leq d$ then

$$
W^{\prime}=\left\{z \in \mathbb{C} ;|z|>v_{\lambda_{\text {min }}}, v_{0}(\lambda) \text { with } \lambda \in \mathcal{S}^{1} \text { and }|\operatorname{Arg} z|<\frac{n}{m} \pi\right\} .
$$

- If $n>d$ then

$$
W^{\prime}=\left\{z \in \mathbb{C} ;|z|>v_{\lambda_{\min }}, v_{0}(\lambda) \text { with } \lambda \in \mathcal{S}^{1} \text { and }|\operatorname{Arg} z|<\frac{d-1}{n-1} \frac{n}{m} \pi\right\} .
$$


The precise values of $v_{\lambda_{\min }}$ are computed in Equation (3.4). The function $G(\lambda)=v_{\lambda}=(m / n)(d \lambda / n)^{\frac{n}{m}}$, see Equation (2.2), takes the subset $\mathcal{O}^{\prime}$ of the parameter plane univalently onto the open set $W^{\prime}$ in the dynamical plane.

Also, for fixed $\theta$, the function $\lambda \rightarrow \xi_{\lambda}^{k}(\theta)$ is analytic on $\mathcal{O}^{\prime}$ and we claim that for each $\theta$ such that $|\theta| \leq 2 \pi d n^{k-1} / m$, then the set of points $\xi_{\lambda}^{k}(\theta)$ lies inside a compact set in $W^{\prime}$. To see the claim we deal first with the arguments of the boundaries. We notice that in Proposition 3.10 we precisely computed the arguments of the set of points in $\xi_{\lambda}^{k}(\theta)$ for $|\theta| \leq 2 \pi d n^{k-1} / m$. Thus, if $z$ is $\xi_{\lambda}^{k}(\theta)$ for $|\theta| \leq 2 \pi d n^{k-1} / m$ it follows that (see Proposition 3.10),

- If $d \geq n$ with $n \geq 5$ then $|\operatorname{Arg} z|<4 \pi / m<n \pi / m$.

- If $n>d$ with $n \geq 5$ then $|\operatorname{Arg} z|<(n / m)(d-1) /(n-1)$.

We notice that by definition $\xi_{\lambda}^{k}(\theta)$ lies inside the open set bounded by $\Gamma_{\lambda}(\theta)$. Hence, for each $\theta$ with $|\theta|<2 \pi d n^{k-1} / m$, the set of points $\xi_{\lambda}^{k}(\theta)$ lies inside a compact set in $W^{\prime}$. Hence we may consider the composition $Q(\lambda)=G^{-1}\left(\xi_{\lambda}^{k}(\theta)\right)$ acting on a region of the parameter space. As a function of $\lambda, Q$ is analytic and maps the simply connected region $\mathcal{O}^{\prime}$ inside itself. By the Schwarz Lemma, $Q$ has a unique fixed point in this set or on its boundary. But the fixed point cannot lie at $\lambda=0$ since 0 is surrounded by the McMullen domain so that the curves $\xi_{\lambda}^{k}$ are bounded away from the origin. Hence there must be a unique fixed point in the interior of $\mathcal{O}^{\prime}$. This fixed point is $\lambda_{\theta, k}$.

Notice that for each $k$, the fixed point $\lambda_{\theta, k}$ varies continuously with $\theta$, so $\theta \rightarrow \lambda_{\theta, k}$ is a curve in the parameter plane. The following proposition identifies the specific values of $\lambda_{\theta, k}$ corresponding to the special cases considered earlier.

In the case $\theta=0$, the parameters $\lambda_{k+1} \in \mathbb{R}^{+}$and $\xi_{\lambda_{k+1}}^{k}(0)=v_{\lambda_{k+1}}$, so that $\lambda_{0, k}=\lambda_{k+1}$. When $\theta=\alpha_{k}$, if $a=\lambda_{k+1}$, it follows that $\xi_{a \omega}^{k}\left(\alpha_{k}\right)=v_{0}$. So $\lambda_{\theta, k}=\omega \lambda_{k+1}$. We write this result as a proposition for future reference.

Proposition 3.13 When $\theta=0$ and $k \geq 1$, the parameter values $\lambda_{0, k}$ are given by the parameters $\lambda_{k+1} \in \mathbb{R}^{+}$. When $\theta=\alpha_{k}$, then $\lambda_{\theta, k}$ is given by $\omega \lambda_{k+1}$ on the symmetry line $\omega \cdot \mathbb{R}^{+}$.

The parameters in Proposition 3.13 are the unique parameters, on the corresponding lines in parameter space, for which the orbit of the second 
iterate of the appropriate critical point monotonically decreases along the corresponding line for $k-1$ iterations, before returning to itself and becoming periodic.

So the curve $\theta \rightarrow \lambda_{\theta, k}$ meets each of these symmetry lines only once. Hence the portion of this curve defined for $0 \leq \theta \leq \alpha_{k}$ either lies outside the sector

$$
0 \leq \operatorname{Arg} \lambda \leq \frac{2 \pi}{n-1}
$$

for all values of $\theta$, or else this entire curve lies inside the sector. But the former cannot occur since this would imply that some $\lambda_{\theta, k}$ would lie in $\mathbb{R}^{-}$, contradicting the fact that each $\lambda_{\theta, k}$ lies in $\mathcal{O}^{\prime}$. Hence the portion of the curve $\lambda_{\theta, k}$ defined for $0 \leq \theta \leq \alpha_{k}$ is a continuous arc connecting $\theta=0$ and $\theta=2 \pi /(n-1)$. It then follows by the $(n-1)$-fold symmetry that, for each $k \geq 1$, then $\lambda_{\theta, k}$ is a simple closed curve in parameter $\lambda$-plane which is periodic of period

$$
\begin{aligned}
(n-1) \alpha_{k} & =(n-1)\left(\frac{2 \pi}{m}\left(1+n+\cdots n^{k-1}-d n^{k-1}\right)\right) \\
& =\frac{2 \pi}{m}\left(n^{k}-1-d n^{k}+d n^{k-1}\right) .
\end{aligned}
$$

We therefore define the ring $\mathcal{S}^{k+1}$ to be the simple closed curve $\theta \rightarrow \lambda_{\theta, k}$. That is, $\mathcal{S}^{k+1}$ consists of parameter values for which the critical orbit has the following behavior:

1. the critical values lie inside the curve $\gamma_{\lambda}^{0}$;

2. $F_{\lambda}^{2}\left(c_{\lambda}\right)$ lies on $\gamma_{\lambda}^{k-1}$

3. subsequent iterates decrease through the $\gamma_{\lambda}^{j}$ until, at the $k^{\text {th }}$ iterate, the critical orbit lands back on the curve $\gamma_{\lambda}^{0}$.

We have shown:

Lemma 3.14 When $n, d \geq 5$ and for $k \geq 1$, the ring $\mathcal{S}^{k+1}$ in parameter space is a simple closed curve that is parameterized by $\theta \rightarrow \lambda_{\theta, k}$ and is periodic of period

$$
\frac{2 \pi}{m}\left(n^{k-1} d(n-1)-n^{k}+1\right)
$$


These curves accumulate on the boundary of the McMullen domain. Moreover, there are precisely $\tau_{k}^{n, d}=d n^{k-2}(n-1)-n^{k-1}+1$ parameters along $\mathcal{S}^{k+1}$ that are superstable parameters and the same number of centers of Sierpinski holes.

This concludes the proof of our main result Theorem A for $n, d \geq 5$.

\section{Discussion}

For the case when $n$ or $d$ are not larger than four, the proof does not work because the function $G(\lambda)$ does not cover enough of the curves $\gamma_{\lambda}^{k}$ to find the fixed point $\lambda_{\theta, k}$, see [3]. We believe that the result still holds for all these cases, as long as $1 / n+1 / d<1$. However, we would need to use $G(\lambda)=F_{\lambda}^{q}\left(v_{\lambda}\right)$ for some $q \in \mathbb{N}$, with $q>1$, and then estimate the location of $\partial B_{\lambda}$ and $\mathcal{M}$ to prove that the function $G$ has the required properties, for each particular case. Therefore, we leave these cases for future work.

\section{Acknowledgements}

Sebastian M. Marotta would like to thank Prof. Bob Devaney for his help and encouragement. Dr. Jang worked on this paper during a visit of the CAS supported by the TWAS-UNESCO Associateship Scheme. Antonio Garijo has been partially supported by MINECO-AEI grants MTM-2017-86795-C32-P.

\section{References}

[1] C. McMullen. Automorphisms of rational maps. Holomorphic Functions and Moduli. Vol 1. Math. Sci. Res. Inst. Pub. 10. Springer, New York (1988), pp. 54-56.

[2] R.L. Devaney, D. Look and D. Uminsky. The Escape Trichotomy for Singularly Perturbed Rational Maps. Indiana University Mathematics Journal 54 (2005), pp. 1621-1634.

[3] R.L. Devaney and S.M. Marotta. The McMullen Domain: Rings Around the Boundary. Transactions of the American Mathematical Society 359 (2007), pp. 3251-3273. 
[4] R.L. Devaney. Baby Mandelbrot Sets Adorned with Halos in Families of Rational Maps. Contemporary Mathematics 396 (2006), pp. 37-50.

[5] P. Roesch. On Captures for the Family $f_{\lambda}(z)=z^{2}+\lambda / z^{2}$. In Dynamics on the Riemann Sphere. European Mathematical Society, Zürich, 2006, pp. 121-130.

[6] H. Jang, Y. So, and S.M. Marotta. Generalized Baby Mandelbrot Sets Adorned with Halos in Families of Rational Maps. Journal of Difference Equations and Applications 23 (3) (2017), pp. 503-520.

[7] N. Steinmetz. Sierpiński curve Julia sets of rational maps. Computational Methods and Function Theory 6 (2006), pp. 317-327.

[8] N. Steinmetz. On the Dynamics of the McMullen Family $R(z)=$ $z^{m}+\lambda / z^{l}$. Conformal Geometry \& Dynamics 10 (2006), pp. 159-183.

[9] R.L. Devaney, K. Josić and Y. Shapiro. Singular Perturbations of Quadratic Maps. International Journal of Bifurcation and Chaos 14 (1) (2004), pp. 161-169.

[10] P. Blanchard, R.L. Devaney, D. M. Look, P. Seal and Y. Shapiro. Sierpinski curve Julia sets and singular perturbations of complex polynomials. Ergodic Theory \& Dynamical Systems 25 (2005), pp. 10471055 .

[11] R.L. Devaney. Structure of the McMullen domain in the Parameter Planes for Rational Maps. Fundamenta Mathematicae 185 (2005), pp. 267-285.

[12] R.L. Devaney, M. Holzer, D. Look, M. Moreno Rocha and D. Uminsky. Singular Perturbations of $z^{n}$. Transcendental Dynamics and Complex Analysis. P. Rippon and G. Stallard Editors. Cambridge University Press, New York, 2008, pp. 111-137.

[13] R.L. Devaney. The McMullen Domain: Satellite Mandelbrot Sets and Sierpiński Holes. Conformal Geometry and Dynamics 11 (2007), pp. 164-190.

[14] F. Çilingir, R.L. Devaney and E.R. Russell. Extending external rays throughout the Julia sets of rational maps. Journal of Fixed Point Theory And Applications 7 (2010), pp. 223-240. 
[15] R.L. Devaney. Dynamics of $z^{n}+\lambda / z^{n}$; Why the Case $n=2$ is Crazy. Conformal Dynamics and Hyperbolic Geometry. Contemporary Mathematics. AMS 573 (2012), pp. 49-65.

[16] R.L. Devaney and E.R. Russell. Connectivity of Julia Sets for Singularly Perturbed Rational Maps. Chaos, CNN, Memristors and Beyond. World Scientific, Singapore, 2013, pp. 239-245.

[17] R.L. Devaney and K. Pilgrim. Dynamic Classification of Escape Time Sierpinski Curve Julia Sets. Fundamenta Mathematicae 202 (2009), pp. 181-198.

[18] R.L. Devaney. Singular Perturbations of Complex Analytic Dynamical Systems. Nonlinear Dynamics and Chaos: Advances and Perspectives. Springer-Verlag, Berlin, 2010, pp. 13-29.

[19] R.L. Devaney. Singular Perturbations of Complex Polynomials. Bulleting of the American Mathematical Society 50 (2013), pp. 391-429.

[20] R.L. Devaney. A Mandelpinski Maze for Rational Maps of the Form $z^{n}+\lambda / z^{d}$. Indagationes Mathematicae 27 (2016), pp. 1042-1058.

[21] R.L. Devaney. Mandelpinski Spokes in the Parameter Planes for Rational Maps. Journal of Difference Equations and Applications 22 (2016), pp. 330-342.

[22] R.L. Devaney. Mandelpinski Structures in the Parameter Planes of Rational Maps. Proceedings of the Oxtoby Centennial Conference, AMS Contemporary Math Series 678 (2016), pp. 133-150.

[23] J. Milnor. Dynamics in one complex variable. 2nd Edition, ViewegVerlag, Göttingen, 2000.

[24] A.F. Beardon. Iteration of rational functions. Springer-Verlag, New York, 1991.

[25] L. Carleson, T.W. Gamelin. Complex dynamics. Springer-Verlag, New York, 1993.

[26] S. Morosawa, Y. Nishimura, M. Taniguchi and T. Ueda. Holomorphic Dynamics. Cambridge University Press, Cambridge, 2000. 
[27] N. Steinmetz. Rational Iteration: Complex Analytic Dynamical Systems. De Gruyter Studies in Mathematics 16. Cambridge University Press, Berlin, 1993.

[28] G. T. Whyburn. Topological Characterization of the Sierpinski Curve. Fundamenta Mathematicae 45 (1958), pp. 320-324.

[29] P. Blanchard, F. Çilingir, D. Cuzzocreo, R.L. Devaney, D. Look and E.D. Russell. Checkerboard Julia Sets for Rational Maps. International Journal of Bifurcation and Chaos 23 (2013), pp. 48-60.

[30] R.L. Devaney. A Myriad of Sierpinski Curve Julia Sets. Difference Equations, Special Functions, and Orthogonal Polynomials. World Scientific, Singapore, 2007, pp. 131-148.

[31] R.L. Devaney and D.M. Look. Buried Sierpiński Curve Julia Sets. Discrete and Continuous Dynamical Systems 13 (2005), pp. 10351046 .

[32] R.L. Devaney and D.M. Look. A Criterion for Sierpiński Curve Julia Sets. Topology Proceedings 30 (2006), pp. 163-179.

[33] H.G. Jang. The Rings Around the McMullen Domain in Families of Rational Maps $F_{\lambda}(z)=z^{n}+\lambda / z^{m}$. Manuscript, 2014. 